REVESCO. Revista de Estudios Cooperativos

ISSN: $1885-8031$

戸 EDICIONES

http://dx.doi.org/10.5209/REVE.62811

\title{
Cooperativismo agroalimentario en Europa. Dimensión, gobernanza y análisis BCG de las sociedades cooperativas TOP25 de la UE-28 y TOP10 en España
}

\author{
Antonio Colom Gorgues ${ }^{1}$, Pilar Cos Sánchez ${ }^{2}$ y Rosa M. Florensa Guiu ${ }^{3}$
}

Recibido: 13 de julio de 2018 / Aceptado: 19 de octubre de 2018

Resumen. Este trabajo tiene por objeto analizar la situación del cooperativismo agroalimentario en Europa, para identificar las estrategias comunes aparecidas en los escenarios europeos y analizar la dinámica hacia el futuro en este siglo XXI. Un pilar fundamental de este trabajo es, por un lado, analizar la dimensión y los modelos de sociedades cooperativas agroalimentarias europeas, por otro, el dinamismo y posición empresarial de las mismas, efectuando un análisis según la matriz portafolio del Boston Consulting Group (BCG), y finalizando con el estudio y análisis de las formas o modelos de gobierno interno.

Las nuevas formas de gobernanza interna aparecidas dentro de un contexto integrador buscando mayor grado de eficacia y eficiencia, y para ello con tendencia hacia una mejora organizativa y mayor dimensión estructural y de comercio internacional, son compatibles con estas formas jurídicas de sociedades cooperativas, al igual que lo son las actuaciones integradas de estas, con otras formas jurídicas de cariz mercantilista, a veces denominados modelos híbridos, pero que abogan por la utilidad y ventajas de la asunción y aplicación de los principios y valores cooperativos.

La empresa societaria cooperativa agroalimentaria ha demostrado en su evolución en el tiempo, ser un modelo capaz de adaptarse a los cambios y dinámica global de la economía y la sociedad, integrando en su actividad cooperativizada elementos concretos de las buenas prácticas para la obtención de buenos resultados sociales y económicos, con el compromiso de respeto y conservación medioambiental.

Palabras clave: Dimensión cooperativa; Matriz portafolio Boston Consulting Group; OPs; Gobernanza interna; Modelos híbridos.

Claves Econlit: Q13; Q17; Q18.

[en] Agri-food cooperatives in Europe. Dimension, governance and BCG analysis of cooperative societies TOP25 of the EU-28 and TOP10 in Spain

Abstract. This work aims to analyze the situation of agri-food cooperatives in Europe, to identify the common strategies that have appeared in European scenarios and to analyze the dynamics towards the future in this 21 st century. A fundamental pillar of this work is, on the one hand, to analyze the

$1 \quad$ Universidad de Lleida, España

Dirección de correo electrónico: antonio_colom@hotmail.com

2 Universidad de Lleida, España

Dirección de correo electrónico: cspilar@aegern.udl.es

3 Universidad de Lleida, España

Dirección de correo electrónico: rmflorensa@aegern.udl.es 
dimension and the models of European agri-food cooperatives, on the other, the dynamism and business position, carrying out an analysis according to the portfolio matrix of the Boston Consulting Group (BCG), and finalizing with the study and analysis of the forms or models of internal government.

The new forms of internal governance appeared within an integrating context seeking greater efficiency and effectiveness, and for this, with a tendency toward organizational improvement and greater structural dimension and international trade, are compatible with these legal forms of cooperative enterprises. As well as that they are the integrated performances of these, with other legal forms of mercantile aspect, sometimes called hybrid models, but that they plead for the utility and advantages of the assumption and application of cooperative principles and values.

The agri-food cooperative, as enterprise, has shown in its evolution over time, to be a model capable of adapting to changes and global dynamics of the economy and society, integrating in its cooperative activity specific elements of good practices for obtaining good results: social and economic, with the commitment of environmental respect and conservation.

Keywords: Cooperative dimension; Portfolio matrix Boston Consulting Group; POs; Internal Governance; Hybrid models.

Sumario. 1. Introducción. Objeto y estructura del trabajo. 2. Antecedentes y esquema metodológico. 3. Modelos cooperativos agroalimentarios en la Unión Europea. 4. Análisis evolutivo del grupo TOP 25 de las cooperativas agroalimentarias de la UE-28, según la matriz del BCG. 5. Análisis BCG del grupo TOP10 de las cooperativas agroalimentarias de España, años 2015-16. 6. Estudio de la gobernanza cooperativa en Europa y España. 7. Nota final para unas conclusiones. 8. Referencias bibliográficas.

Cómo citar: Colom Gorgues, A., Cos Sánchez, P. y Florensa Guiu, R.M. (2019) Cooperativismo agroalimentario en Europa. Dimensión, gobernanza y análisis BCG de las sociedades cooperativas TOP25 de la UE-28 y TOP10 en España. REVESCO. Revista de Estudios Cooperativos, Primer Cuatrimestre, No 130, pp. 73-98. DOI: 10.5209/REVE.62811.

\section{Introducción. Objeto y estructura del trabajo}

El presente trabajo ha sido realizado con el objetivo general de analizar la situación del cooperativismo agroalimentario en Europa, la dinámica evolutiva de la gobernanza interna y el posicionamiento empresarial dentro de los distintos modelos existentes en Europa. El mundo cooperativo agrario y agroalimentario, recibe en la Unión Europea como se sabe, la influencia de COPA-COGECA, que ya en la actualidad presentan sus alegaciones para la futura PAC post 2020, como que la agricultura ocupa y debe seguir ocupando un lugar fundamental en la Unión Europea como una política fuerte, común y con una financiación apropiada. Los agricultores desempeñan un papel crucial en el suministro de alimentos y participan en la consecución de los objetivos territoriales, medioambientales y sociales. Por ello, el COPA y la COGECA abogan por una PAC común, fuerte, más sostenible y más sencilla. Una política con una visión a largo plazo que apoye a los agricultores a la hora de dar seguridad alimentaria a la UE y de proporcionar alimentos seguros, de calidad y nutritivos producidos de manera sostenible.

Se ha estructurado el trabajo en seis apartados. Después de la introducción, en el segundo se explican los antecedentes y se muestra en síntesis el esquema metodológico planteado en el mismo. En el tercero, se tratan de resumir los modelos de cooperativismo agroalimentario en la Unión Europea, viendo su 
evolución y consolidación. En el cuarto, se presenta el análisis evolutivo del grupo TOP25 de las cooperativas agroalimentarias de la Unión Europea-28, según el modelo de la matriz portafolio del Boston Consulting Group (BCG). En el apartado quinto, se estudia y analiza la evolución actual del sistema de gobernanza interna cooperativa en la Unión Europea y en España. En el sexto y último apartado se termina el artículo con una nota final para las conclusiones.

\section{Antecedentes y esquema metodológico}

Este trabajo supone una parte del proceso de investigación de una Tesis Doctoral, que se desarrolla en el campo de las Organizaciones de Productores (OPs) y el Cooperativismo Agroalimentario, para tratar de ver su evolución y estado actual en Europa y en España, y sobre los planteamientos estratégicos hacia el futuro.

También se señalan como antecedentes las colaboraciones realizadas en el ámbito cooperativo con CEGEA de la Universidad Politécnica de Valencia (UPV), citando la realización de Workshops Internacionales en la UPV, Jornadas y Workshop en la Universidad de Lleida, colaboraciones en capítulos de libros sobre la estructura y gobernabilidad de las cooperativas agroalimentarias, realización de un informe sobre una Tesis Doctoral, e intercambio de datos e información.

Como punto de partida de este trabajo, se ha considerado la diferenciación de modelos cooperativos en Europa según Baamonde (2009), analizando la evolución actual según los datos de COGECA (2014) y comprobando la consolidación de dichos modelos. Se han estudiado y analizado las ratios de Facturación/Cooperativa, Facturación/Socio, Número de Trabajadores/Cooperativa y los números índice comparativos sobre 100.

Se ha efectuado a continuación el análisis dinámico bicampaña del posicionamiento de las principales cooperativas agroalimentarias TOP25 de la Unión Europea-28, siguiendo el modelo de la matriz portafolio del Boston Consulting Group (BGC) de Henderson (1973), analizando la evolución de la posición empresarial en estas dos campañas 2011-2013.

Finalmente, y a partir de una recopilación de artículos sobre la evolución de la estructura, el marco organizativo y la gobernanza interna cooperativa, referenciando los trabajos de Arcas (2001), Arcas y Hernández (2003), Arcas et al. (2000, 2011a, 2011b), Baamonde (2009), Bijman et al. (2013, 2014a, 2014b), Borguen (2004), Borjabad (2001), Carnicer (2017), Chaddad e Iliopoulos (2013), Colom y Mallada (2010), Colom (2015), Hansen et al. (2002), Juárez et al. (2012), Juliá et al. (2004, 2011, 2013), Meliá et al. (2016), Mínguez et al. (2010), Puentes et al. (2009), Salazar y Galve (2009), Valentinov (2004), Volkers y Lees (1996), se han analizado los tipos de gobierno interno actuales en la Unión Europea y España.

\section{Modelos cooperativos agroalimentarios en la Unión Europea}

Las sociedades cooperativas agrarias en la Unión Europea son un importante factor socioeconómico en el ámbito económico y social en general: Sostienen una cuota de mercado de más del 50 \% del suministro de insumos agrícolas; y realizan más 
del $60 \%$ de cuota de mercado en las actividades de recolección, transformación y comercialización de los productos agrícolas y agroalimentarios.

Hay una gran diferencia entre el tamaño o dimensión, facturación y ratios de eficiencia según países. Años atrás, Baamonde (2009) propuso 3 modelos según tres típicas áreas geográficas europeas (ver tabla 1):

1. Modelo Nórdico: Comprende los países del Norte de Europa como Dinamarca, Holanda, Suecia, Finlandia e Irlanda.

2. Modelo Centroeuropeo: Comprende países centroeuropeos como Francia, Alemania y Bélgica.

3. Modelo Mediterráneo: Comprende países del Sur de Europa como España, Italia y Grecia.

Si tenemos en cuenta una serie de indicadores de tamaño y eficiencia como a: Facturación / Cooperativa; b: Facturación / Socio; c: Empleo / Cooperativa; y d: Facturación / Empleado, diremos que los datos Promedio en Europa son (entre paréntesis, primero los datos de España y después los máximos de los modelos centroeuropeo y nórdico):

a: Facturación/Cooperativa $=10,90$ Miles Euros/Cooperativa (España 4,20; Francia 19,14; Dinamarca 1.346,13)

b: Facturación / Socio = 39 Miles Euros / Socio (España 17; Francia 116; Holanda 316)

c: Empleo / Cooperativa $=30$ Empleados / Cooperativa (España 23; Bélgica 56; Dinamarca 2.500)

d: Facturación / Empleado =0,37 Miles de Euro / Empleado (España 0,19; Francia 0,45; Holanda 0,76)

Tabla. 1. Modelos Cooperativos Agroalimentarios en la Unión Europea: Nórdico, Centroeuropeo y Mediterráneo

\begin{tabular}{|c|c|c|c|c|c|c|c|c|c|}
\hline $\begin{array}{l}\text { Modelos } \\
\text { Cooperativos }\end{array}$ & Países & $\begin{array}{l}\text { Fac./Coop. } \\
\text { Miles } €\end{array}$ & $\begin{array}{l}\text { Índice/ } \\
100\end{array}$ & $\begin{array}{l}\text { Fac./Socio } \\
\text { Miles } €\end{array}$ & $\begin{array}{l}\text { Índice/ } \\
100\end{array}$ & $\begin{array}{l}\text { Empleo/Coop. } \\
\mathrm{N}^{\mathrm{o}} \text { trabajad. }\end{array}$ & $\begin{array}{l}\text { Índice } \\
/ 100\end{array}$ & $\begin{array}{l}\text { Fac./trab. } \\
\text { Miles } €\end{array}$ & $\begin{array}{l}\text { Índice/ } \\
100 \\
\end{array}$ \\
\hline \multirow{5}{*}{$\begin{array}{l}\text { MODELO } \\
\text { NÓRDICO }\end{array}$} & Dinamarca & $1.346,13$ & 100,00 & 231 & 73,10 & 2.500 & $\begin{array}{l}100,0 \\
0\end{array}$ & 0,54 & 71,05 \\
\hline & Holanda & $1.026,36$ & 76,25 & 316 & 100,00 & 1.355 & 54,20 & 0,76 & 100,00 \\
\hline & Suecia & 370,59 & 27,53 & 42 & 13,29 & 882 & 35,28 & 0,42 & 55,26 \\
\hline & Finlandia & 277,08 & 20,58 & 62 & 19,62 & 942 & 37,68 & 0,29 & 38,16 \\
\hline & Irlanda & 125,25 & 9,30 & 63 & 19,94 & 354 & 14,16 & -- & -- \\
\hline \multirow{3}{*}{$\begin{array}{l}\text { MODELO } \\
\text { CENTRO - } \\
\text { EUROPEO }\end{array}$} & Francia & 19,14 & 1,42 & 116 & 36,71 & 43 & 1,72 & 0,45 & 59,21 \\
\hline & Alemania & 11,26 & 0,84 & 16 & 5,06 & 37 & 1,48 & 0,31 & 40,79 \\
\hline & Bélgica & 7,75 & 0,58 & 42 & 13,29 & 56 & 2,24 & 0,14 & 18,42 \\
\hline \multirow{4}{*}{$\begin{array}{l}\text { MODELO } \\
\text { MEDITE- } \\
\text { RRÁNEO }\end{array}$} & Italia & 5,27 & 0,39 & 35 & 11,08 & 17 & 0,68 & 0,32 & 42,11 \\
\hline & España & 4,20 & 0,31 & 17 & 5,38 & 23 & 0,92 & 0,19 & 25,00 \\
\hline & Grecia & 0,16 & 0,01 & 1 & 0,32 & 4 & 0,16 & 0,04 & 5,26 \\
\hline & $\begin{array}{l}\text { Promedio } \\
\text { UE }\end{array}$ & 10,90 & 0,81 & 39 & 12,34 & 30 & 1,20 & 0,37 & 48,68 \\
\hline
\end{tabular}

Fuente: Baamonde (2009), y elaboración propia 
Las diferencias entre modelos cooperativistas en Europa son notorias y palpables como vemos en la tabla1:

- El Modelo Mediterráneo asume la corta dimensión y peor eficiencia manteniendo las ratios anteriores por debajo de los promedios europeos, y corresponde al grupo donde se encuentra España.

- En el Modelo Centroeuropeo de dimensión cooperativa intermedia y mayor que las del grupo anterior, las ratios en general son más altas con alguna excepción, y

En cambio, en el Modelo Nórdico, vemos las sociedades cooperativas de mayor dimensión y las mayores ratios de eficiencia. Destacan claramente los países nórdicos de Dinamarca y Holanda donde es conocida por todos, la importancia de las sociedades cooperativas, su dimensión, eficiencia y la magnitud de negocio.

Tabla. 2. Datos generales y cooperativos de los países de la Unión Europea-28

\begin{tabular}{|c|c|c|c|c|c|c|c|c|c|c|c|c|c|c|}
\hline $\mathrm{N}^{\circ}$ & $\begin{array}{l}\text { Países } \\
\text { Miembros }\end{array}$ & $\begin{array}{l}\text { Total } \\
\text { Área, } \\
\text { km2 }\end{array}$ & $\begin{array}{l}\text { Población, } \\
000 \\
\text { habitantes }\end{array}$ & $\begin{array}{l}\text { SAU, } \\
000 \text { ha }\end{array}$ & $\begin{array}{l}\text { SAU } \\
\text { por } \\
\text { explot. } \\
\text { ha } \\
\end{array}$ & $\begin{array}{l}\text { Empleo } \\
\text { agrario, } \\
\%\end{array}$ & $\begin{array}{l}\text { VAB } \\
A / P I \\
B\end{array}$ & $\begin{array}{l}\text { Output } \\
\text { M€ Agro- } \\
\text { industria }\end{array}$ & $\begin{array}{l}N^{0} \text { Total } \\
\text { de Coop. } \\
2014\end{array}$ & $\begin{array}{l}N^{0} \text { Total } \\
\text { de } \\
\text { Socios }\end{array}$ & $\begin{array}{l}\text { Cifra } \\
\text { de } \\
\text { Ventas } \\
\text { M€ } \\
\end{array}$ & $\begin{array}{l}\% \text { Cifra } \\
\text { de } \\
\text { Ventas }\end{array}$ & $\begin{array}{l}\text { Ventas } / \mathrm{N}^{\circ} \\
\text { de Coop, } \\
\mathrm{M} \in / \text { Coop }\end{array}$ & $\begin{array}{l}\text { Ventas } \\
\text { por Socio } \\
000 € / \text { so. }\end{array}$ \\
\hline 1 & Alemania & 357.127 & 81.844 & 16.704 & 4.9 & 1.6 & 0.6 & 54.578 & 2.400 & 1.440 .60 & 67.502 & $19,43 \%$ & 28,13 & 46,86 \\
\hline 2 & Austria & 83.870 & 8.408 & 2.878 & 2.9 & 4.5 & 1.0 & 7.245 & 217 & 306.300 & 8.475 & $2,44 \%$ & 39,06 & 27,67 \\
\hline 3 & Bélgica & 30.528 & 11.095 & 1.358 & 8.2 & 1.3 & 0.6 & 8.545 & 301 & $:$ & 3.257 & $0,94 \%$ & 10,82 & \\
\hline 4 & Bulgaria & 111.001 & 7.327 & 4.476 & 1.6 & 18.9 & 4.2 & 4.424 & 900 & : & : & & & \\
\hline 5 & Chipre & 9.250 & 862 & 118 & 7.3 & 3.6 & 1.9 & 720 & 14 & 24.917 & 62 & $0,02 \%$ & 4,43 & 2,49 \\
\hline 6 & Croacia & 56.590 & 4.276 & 1.331 & 3.0 & $:$ & 2.7 & 2.780 & 613 & 10.734 & 167 & $0,05 \%$ & 0,27 & 15,56 \\
\hline 7 & Dinamarca & 43.098 & 5.581 & 2.647 & 2.1 & 2.4 & 1.5 & 11.873 & 28 & 45.710 & 25.009 & $7,20 \%$ & 893,18 & 547,12 \\
\hline 8 & Eslovaquia & 49.036 & 5.404 & 1.896 & 2.9 & 3.1 & 0.8 & 2.397 & 597 & $:$ & 1.151 & $0,33 \%$ & 1,93 & \\
\hline 9 & Eslovenia & 20.270 & 2.055 & 483 & 4.3 & 8.3 & 1.1 & 1.149 & 368 & 16.539 & 705 & $0,20 \%$ & 1,92 & 42,63 \\
\hline 10 & España & 505.365 & 46.818 & 23.753 & 2.0 & 4.2 & 2.1 & 42.191 & 3.844 & 1.179.32 & 25.696 & $7,40 \%$ & 6,68 & 21,79 \\
\hline 11 & Estonia & 45.227 & 1.334 & 941 & 1.4 & 4.7 & 2.1 & 898 & 21 & 2.036 & 512 & $0,15 \%$ & 24,38 & 251,47 \\
\hline 12 & Finlandia & 338.420 & 5.401 & 2.291 & 2.4 & 4.6 & 0.9 & 5.032 & 35 & 170.776 & 13.225 & $3,81 \%$ & 377,86 & 77,44 \\
\hline 13 & Francia & 551.695 & 65.800 & 27.837 & 2.3 & 2.8 & 1.6 & 77.353 & 2.400 & 858.000 & 84.350 & $24,28 \%$ & 35,15 & 98,31 \\
\hline 14 & Grecia & 131.982 & 11.123 & 5.178 & 2.1 & 12.2 & 2.8 & 10.752 & 550 & : & 711 & $0,20 \%$ & 1,29 & \\
\hline 15 & Hungría & 93.034 & 9.932 & 4.686 & 2.1 & 7.4 & 2.7 & 7.514 & 1.116 & 31.544 & 1.058 & $0,30 \%$ & 0,95 & 33,54 \\
\hline 16 & Irlanda & 70.285 & 4.583 & 4.991 & 0.9 & 4.7 & 1.1 & 7.049 & 75 & 201.684 & 14.149 & $4,07 \%$ & 188,65 & 70,15 \\
\hline 17 & Italia & 301.323 & 60.821 & 12.856 & 4.7 & 3.8 & 1.6 & 48.632 & 5.834 & 863.323 & 34.362 & $9,89 \%$ & 5,89 & 39,80 \\
\hline 18 & Letonia & 64.559 & 2.045 & 1.796 & 1.1 & 7.9 & 1.4 & 1.323 & 49 & : & 1.111 & $0,32 \%$ & 22,67 & \\
\hline 19 & Lituania & 65.300 & 3.004 & 2.743 & 1.1 & 8.8 & 3.5 & 2.973 & 402 & 12.900 & 714 & $0,21 \%$ & 1,78 & 55,35 \\
\hline 20 & Luxemburgo & 2.586 & 525 & 131 & 4.0 & 1.1 & 0.3 & 397 & 55 & : & : & & & \\
\hline 21 & Malta & 316 & 418 & 11 & 36.5 & 3.2 & 0.8 & 128 & 18 & 1.815 & 204 & $0,06 \%$ & 11,33 & 112,40 \\
\hline 22 & Países Bajos & 37.355 & 16.730 & 1.872 & 8.9 & 2.5 & 1.4 & 26.268 & 215 & 140.000 & 32.000 & $9,21 \%$ & 148,84 & 228,57 \\
\hline 23 & Polonia & 312.680 & 38.538 & 14.447 & 2.7 & 12.6 & 2.4 & 23.198 & 136 & : & 15.311 & $4,41 \%$ & 112,58 & \\
\hline
\end{tabular}




\begin{tabular}{|l|l|l|l|l|l|l|l|l|l|l|l|l|l|l|}
24 & Portugal & 91.909 & 10.542 & 3.668 & 2.9 & 11.0 & 1.3 & 6.466 & 735 & $:$ & 2.437 & $0,70 \%$ & 3,32 & \\
\hline 25 & Reino Unido & 244.101 & 63.495 & 16.882 & 3.8 & 1.2 & 0.5 & 29.257 & 200 & 138.021 & 6.207 & $1,79 \%$ & 31,04 & 44,97 \\
\hline 26 & Rep. Checa & 78.866 & 10.505 & 3.484 & 3.0 & 3.3 & 0.9 & 4.866 & 548 & 524 & 1.327 & $0,38 \%$ & 2,42 & 2532,44 \\
\hline 27 & Rumanía & 238.391 & 20.096 & 13.306 & 1.5 & 30.6 & 4.7 & 14.410 & 68 & $:$ & 204 & $0,06 \%$ & 3,00 & \\
\hline 28 & Suecia & 447.420 & 9.483 & 3.066 & 3.1 & 2.1 & 0.5 & 6.429 & 30 & 160.350 & 7.438 & $2,14 \%$ & 247,93 & 46,39 \\
\hline & TOTAL & 4.380 .98 & 509.585 & 177.84 & - & - & - & $\mathbf{4 1 0 . 8 5 8}$ & $\mathbf{2 1 . 7 6 9}$ & $\mathbf{6 . 1 7 2 . 7 4}$ & $\mathbf{3 4 7 . 3 4}$ & $\mathbf{1 0 0 \%}$ & - & - \\
\hline
\end{tabular}

Fuente: COGECA, 2015 y elaboración propia

En la tabla 2 se muestran los datos generales sobre los 28 países europeos miembros de la Unión Europea-28, y sobre el conjunto de Cooperativas Agroalimentarias europeas.

Como se puede observar y demostrar, se sigue cumpliendo el criterio de los modelos cooperativos europeos de Baamonde (2009). Con datos de 2014, se muestra:

El mayor tamaño cooperativo con las ratios de Cifra de Ventas/Cooperativa (Millones €/cooperativa) y Ventas/Socio (000€/socio) de las sociedades cooperativas de Dinamarca $(893,18$ y 547,12$)$, Finlandia $(377,86$ y 77,44$)$, Suecia $(247,93$ y 46,39) y Holanda $(148,84$ y 228,57$)$ dentro del Modelo Nórdico;

- Se muestran, en una posición intermedia, las ratios de Austria $(39,06$ y $27,67)$, Francia $(35,15$ y 98,31$)$, Alemania $(28,13$ y 46,86) y Estonia $(24,38$ y 251,47) dentro del Modelo Centroeuropeo; y

- Finalmente se observan las ratios más reducidas de España $(6,68$ y $21,79)$, Italia $(5,89$ y 39,80), Malta $(11,33$ y 112,40$)$, Chipre $(4,43$ y 2,49$)$ y Croacia $(0,27$ y 15,56) dentro del Modelo Mediterráneo.

O sea, se comprueba la actualización y vigencia de los tres modelos citados.

\section{Análisis evolutivo del grupo TOP25 de las cooperativas agroalimentarias de la UE-28, según la matriz del BCG}

En este apartado, y a través de los datos de la tabla 3, se realiza un análisis evolutivo bicampaña (2011-2013) de la posición empresarial según la Matriz del Boston Consulting Group (BCG) de Bruce Henderson (Henderson, 1973, Colom, 2015) del grupo TOP25 de las mayores cooperativas agroalimentarias de la UE-28.

A manera de marco teórico resumido, la Matriz Portafolio del BCG, es una herramienta para el análisis estratégico empresarial (también aplicable para productos y unidades estratégicas de negocios), analizando las dos variables siguientes:

La Cuota Relativa de mercado de la sociedad cooperativa, para expresar su posición competitiva en el mercado, en un año o campaña del año n. Esta variable está relacionada con la capacidad de la empresa de generar CashFlow. 
- La Tasa de Crecimiento en el mercado para la sociedad cooperativa, desde la campaña anterior a la actual ([cuota campaña del año $\mathrm{n}$ - cuota campaña del año n-1] / cuota campaña del año n-1). Esta otra variable induce a la posibilidad de necesitar o aplicar Cash-Flow (para inversión, $\mathrm{I}+\mathrm{D}+\mathrm{T}+\mathrm{i}$, crecimiento empresarial).

El \% de Cuota Relativa de mercado se coloca en el eje horizontal y el \% de Tasa de Crecimiento en el mercado en el vertical de los ejes coordenados. Ambos ejes se dividen en dos zonas ALTA y BAJA cuya frontera es la media de ambas variables. Se forma una cuadrícula de 4 loci (ver la figura 1):

\title{
ALTA-ALTO: Estrellas:
}

Alta cuota relativa de mercado y alto \% tasa de crecimiento en el mercado. Se trata de empresas con una muy buena posición que les dará opción a generar buenos niveles de Cash-Flow y posibilidades para inversión, innovación y crecimiento, Es el mejor posicionamiento esperado con posibilidades de buen futuro y buenos resultados empresariales.

\section{BAJA-ALTO: Interrogantes o Dilemas:}

Bajo nivel de cuota relativa de mercado y alto \% de tasa de crecimiento en el mercado. Se trata de un posicionamiento que da lugar a un interrogante (?). La empresa tiene cuota relativa de mercado baja y en cambio está creciendo altamente en cuota de mercado desde la campaña anterior. Tal vez sea el caso de empresas de nueva creación (start-ups, empresas jóvenes, PYMES de nueva creación, etc.) o que han abordado un plan de crecimiento o expansión (empresas en inversión en estructura y aumento productivo), lo cual dará lugar en un futuro a un aumento de la cuota de mercado. Dichas empresas pueden seguir una migración hacia la posición de Estrellas.

\begin{abstract}
ALTA-BAJO: Vacas Lecheras:
Alto nivel de cuota relativa de mercado, pero bajo nivel de crecimiento. Ello posiciona en una condición, tal vez, de empresario especulador, aprovechando su estatus de alta cuota relativa para generar alto nivel de cash-flow, pero en cambio olvida su posible crecimiento (puede ser por falta inversión, innovación, problemas con la demanda, etc.). Esta situación le puede llevar a empeorar su cuota de mercado, a no ser que sea una situación temporal superable.
\end{abstract}

\section{BAJA-BAJO: Perros:}

Bajo nivel tanto de cuota relativa de mercado como de tasa de crecimiento de la cuota. Estas características llevan a clasificar estas empresas como las peor posicionadas, tal vez con una dinámica negativa de pérdida de peso y de competitividad, que pueden abocarlas a una situación de deterioro. No obstante, también tendrían cabida aquí, empresas de pequeña dimensión que tienen una cuota relativa mercado pequeña adecuada a su índole local o regional, atienden a un nicho o pequeño segmento, y no se plantean ninguna estrategia de crecimiento. 
Figura. 1. Esquema del modelo de Matriz del BCG

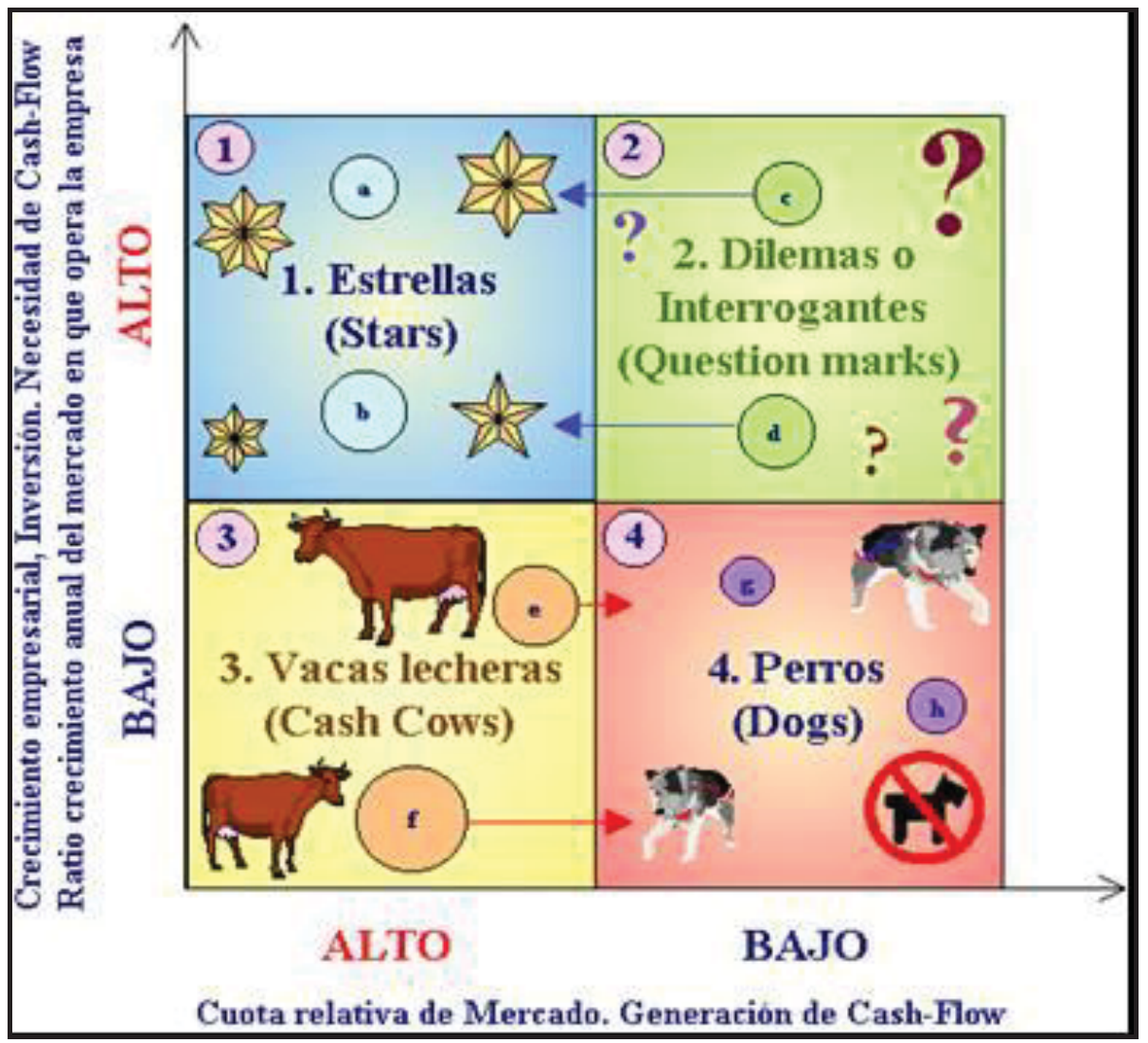

Fuente: Elaboración propia, tomado de Colom (2015)

Los datos de las Cooperativas Agroalimentarias del periodo 2011-2013 han sido procesados con hoja de cálculo Excel, obteniendo los resultados evolutivos que se muestran en la tabla 3 y la figura 2. El proceso de cálculo ha consistido en el cálculo de las dos variables (\% Cuota Relativa de Mercado y \% de Tasa de Crecimiento en el Mercado) para cada una de las sociedades cooperativas TOP25 analizadas en los dos años de referencia. En las dos últimas columnas aparecen los resultados de clasificación y posicionamiento en Estrellas, Interrogantes, Vacas Lecheras y Perros, para los años 2011 y 2012, que dan lugar a los gráficos respectivos. 
Tabla. 3. TOP 25 de las Cooperativas Agroalimentarias UE-28. Datos y resultados para el análisis BCG

\begin{tabular}{|c|c|c|c|c|c|c|c|c|c|c|c|c|}
\hline $\begin{array}{l}\text { Ranking } \\
\text { (2013) }\end{array}$ & Cooperativa & País & $\begin{array}{l}\text { Sector, } \\
\text { productos }\end{array}$ & $\begin{array}{l}\text { Cifra } \\
\text { Ventas } \\
\text { 2011, M€ }\end{array}$ & $\begin{array}{l}\text { Cuota } \\
\text { Relativa } \\
\text { TOP25 } \\
2012, \%\end{array}$ & $\begin{array}{l}\% \text { Cuota } \\
\text { Crecimiento } \\
2012 / 2011\end{array}$ & $\begin{array}{l}\text { Cifra } \\
\text { Ventas } \\
2012 \text {, } \\
\text { M€ }\end{array}$ & $\begin{array}{l}\text { Cuota } \\
\text { Relativa } \\
\text { TOP25 } \\
2013 \%\end{array}$ & $\begin{array}{lr}\% & \text { Cuota } \\
\text { Crecimiento } \\
2012 / 2011\end{array}$ & $\begin{array}{l}\text { Cifra } \\
\text { Ventas } \\
2013 \text {, } \\
\text { M€ }\end{array}$ & $\begin{array}{l}\text { Clase de } \\
\text { empresa } \\
2012\end{array}$ & $\begin{array}{l}\text { Clase de } \\
\text { empresa } \\
2013\end{array}$ \\
\hline 1 & Bay Wa & $\overline{\mathrm{DE}}$ & $\begin{array}{l}\text { Suministros } \\
\text { Agrarios }\end{array}$ & 9.586 & $8,13 \%$ & $9,86 \%$ & 10.531 & $11,25 \%$ & $51,52 \%$ & 15.957 & ES & ES \\
\hline 2 & $\begin{array}{l}\text { Friesland } \\
\text { Campina }\end{array}$ & $\mathrm{NL}$ & Lácteos & 9.626 & $7,96 \%$ & $7,10 \%$ & 10.309 & $8,05 \%$ & $10,76 \%$ & 11.418 & $\mathrm{VL}$ & ES \\
\hline 3 & Arla Foods & DK & Lácteos & 7.384 & $6,53 \%$ & $14,44 \%$ & 8.450 & $6,97 \%$ & $17,01 \%$ & 9.887 & ES & ES \\
\hline 4 & DLG & DK & $\begin{array}{l}\text { Suministros } \\
\text { Agrarios }\end{array}$ & 5.494 & $5,03 \%$ & $18,49 \%$ & 6.510 & $5,60 \%$ & $21,95 \%$ & 7.939 & ES & ES \\
\hline 5 & Danish Crown & DK & Cárnicos & 6.954 & $5,36 \%$ & $-0,20 \%$ & 6.940 & $5,53 \%$ & $13,03 \%$ & 7.844 & $\mathrm{VL}$ & ES \\
\hline 6 & Agravis & $\overline{D E}$ & $\begin{array}{l}\text { Suministros } \\
\text { Agrarios }\end{array}$ & 6.468 & $5,49 \%$ & $9,89 \%$ & 7.108 & $5,29 \%$ & $5,57 \%$ & 7.504 & ES & $\overline{V L}$ \\
\hline 7 & Vion Food & $\overline{N L}$ & Cárnicos & 8.870 & $7,43 \%$ & $8,46 \%$ & 9.620 & $4,96 \%$ & $-26,89 \%$ & 7.033 & $\overline{\mathrm{VL}}$ & $\overline{\mathrm{VL}}$ \\
\hline 8 & InVivo & FR & $\begin{array}{l}\text { Suministros } \\
\text { Agrarios }\end{array}$ & 6.083 & $4,38 \%$ & $-6,81 \%$ & 5.669 & $4,33 \%$ & $8,27 \%$ & 6.138 & $\overline{V L}$ & $\overline{V L}$ \\
\hline 9 & Kerry Group & $\mathrm{IE}$ & Lácteos & 4.700 & $4,52 \%$ & $24,43 \%$ & 5.848 & $4,11 \%$ & $-0,21 \%$ & 5.836 & ES & $\overline{V L}$ \\
\hline 10 & DMK & $\overline{D E}$ & Lácteos & 4.575 & $3,43 \%$ & $-2,99 \%$ & 4.438 & $3,74 \%$ & $19,65 \%$ & 5.310 & $\mathrm{PE}$ & $? ?$ \\
\hline 11 & Metsä Goup & $\mathrm{Fl}$ & Forestal & 5.346 & $3,86 \%$ & $-6,45 \%$ & 5.001 & $3,48 \%$ & $-1,38 \%$ & 4.932 & $\mathrm{PE}$ & $\mathrm{PE}$ \\
\hline 12 & Tereos & FR & $\begin{array}{l}\text { Cullivos, } \\
\text { Azucar }\end{array}$ & 4.409 & $3,89 \%$ & $14,24 \%$ & 5.037 & $3,31 \%$ & $-6,75 \%$ & 4.697 & $? ?$ & $\mathrm{PE}$ \\
\hline 13 & Terrena & FR & $\begin{array}{l}\text { Multi- } \\
\text { produc to }\end{array}$ & 3.871 & $3,46 \%$ & $15,68 \%$ & 4.478 & $3,29 \%$ & $4,22 \%$ & 4.667 & $? ?$ & $\mathrm{PE}$ \\
\hline 14 & Sodiaal & $\overline{F R}$ & Lácteos & 4.021 & $3,41 \%$ & $9,95 \%$ & 4.421 & $3,25 \%$ & $4,41 \%$ & 4.616 & $? ?$ & $\mathrm{PE}$ \\
\hline 15 & FloraHolland & $\overline{N L}$ & Horticultura & 4.130 & $3,31 \%$ & $3,66 \%$ & 4.281 & $3,07 \%$ & $1,61 \%$ & 4.350 & $\mathrm{PE}$ & $\mathrm{PE}$ \\
\hline 16 & VIVESCIA & FR & $\begin{array}{l}\text { Suministros } \\
\text { Cereales }\end{array}$ & 3.483 & $3,08 \%$ & $14,36 \%$ & 3.983 & $2,97 \%$ & $5,67 \%$ & 4.209 & $? ?$ & $\mathrm{PE}$ \\
\hline 17 & Agrial & FR & $\begin{array}{l}\text { Multi- } \\
\text { produc to }\end{array}$ & 2.261 & $2,10 \%$ & $20,08 \%$ & 2.715 & $2,75 \%$ & $43,68 \%$ & 3.901 & $? ?$ & $? ?$ \\
\hline 18 & Lantmännen & SE & $\begin{array}{l}\text { Multi- } \\
\text { producto }\end{array}$ & 4.244 & $3,32 \%$ & $1,37 \%$ & 4.302 & $2,64 \%$ & $-12,83 \%$ & 3.750 & $\mathrm{PE}$ & $\mathrm{PE}$ \\
\hline 19 & Axéréal & FR & $\begin{array}{l}\text { Suministros } \\
\text { Agrarios }\end{array}$ & 3.375 & $2,63 \%$ & $0,74 \%$ & 3.400 & $2,61 \%$ & $9,03 \%$ & 3.707 & $\mathrm{PE}$ & $? ?$ \\
\hline 20 & Danish Agro & DK & $\begin{array}{l}\text { Suministros } \\
\text { Agrarios }\end{array}$ & 2.130 & $1,75 \%$ & $6,24 \%$ & 2.263 & $2,40 \%$ & $50,24 \%$ & 3.400 & $\mathrm{PE}$ & $? ?$ \\
\hline 21 & Glanbia & $\mathrm{IE}$ & Lácteos & 2.735 & $2,35 \%$ & $11,08 \%$ & 3.038 & $2,31 \%$ & $8,03 \%$ & 3.282 & $? ?$ & $\overline{P E}$ \\
\hline 22 & $\begin{array}{l}\text { Agricola tre } \\
\text { valli }\end{array}$ & IT & Cárnicos & 3.056 & $2,39 \%$ & $1,29 \%$ & 3.096 & $2,21 \%$ & $1,28 \%$ & 3.135 & $\mathrm{PE}$ & $\mathrm{PE}$ \\
\hline 23 & Agrana & $\overline{\text { AT }}$ & $\begin{array}{l}\text { Azucar, } \\
\text { Almidón, } \\
\text { Fruta }\end{array}$ & 2.576 & $2,37 \%$ & $18,98 \%$ & 3.065 & $2,14 \%$ & $-0,75 \%$ & 3.042 & $? ?$ & $\mathrm{PE}$ \\
\hline 24 & $\begin{array}{l}\text { RWZ Rhein } \\
\text { Main,Köln }\end{array}$ & $\overline{D E}$ & $\begin{array}{l}\text { Suministros } \\
\text { Agrarios }\end{array}$ & 1.910 & $1,77 \%$ & $20,31 \%$ & 2.298 & $1,89 \%$ & $16,75 \%$ & 2.683 & $? ?$ & $? ?$ \\
\hline \multirow[t]{2}{*}{25} & RWA & $\overline{\mathrm{AT}}$ & $\begin{array}{l}\text { Suministros } \\
\text { Agrarios }\end{array}$ & 2.513 & $2,08 \%$ & $7,28 \%$ & 2.696 & $1,87 \%$ & $-1,45 \%$ & 2.657 & $\mathrm{PE}$ & $\mathrm{PE}$ \\
\hline & & & TOTAL ... & 119.800 & $100,00 \%$ & $221,47 \%$ & 129.497 & $100,00 \%$ & $242,44 \%$ & 141.894 & & \\
\hline
\end{tabular}

Fuente: COGECA, 2015 y elaboración propia (ES=Estrella; ??=Interrogante; VL=Vaca Lechera; PE=Perro) 
Figura. 2. Matriz BGC de las TOP25 Cooperativas Agroalimentarias de la UE-28, años 2012 y $2013^{4}$
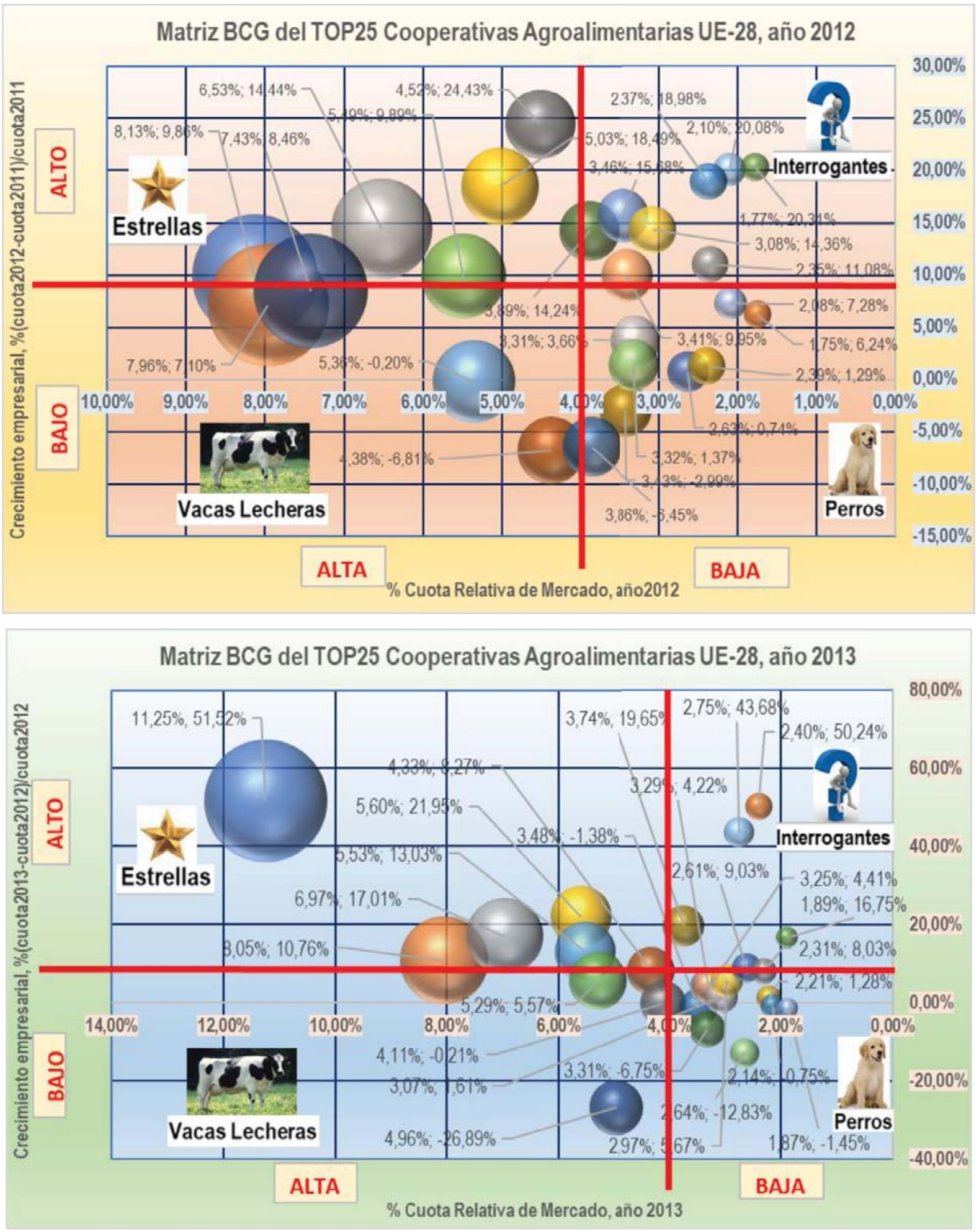

Fuente: Elaboración propia a partir de datos de COGECA, 2015

4 Nota 1: En los gráficos, se muestran para cada una de las TOP25 sociedades cooperativas, los valores de las variables \% Cuota Relativa Mercado y \% Tasa Crecimiento, señalando el centro de cada burbuja cooperativa, que es proporcional a dicha cuota relativa de mercado. Con estos datos se identifica la sociedad cooperativa de que se trata por referencia a los datos de la Tabla 3. 
De los resultados obtenidos aplicando el modelo de Matriz BCG se pueden realizar los siguientes análisis y diagnósticos:

a) Resultados de la Matriz BCG, año 2012:

COOPERATIVAS ESTRELLAS (ALTA \% Cuota Mercado y ALTO \% Tasa Crecimiento): Bay Wa (Alemania, suministros), Arla Foods (Dinamarca, lácteos), DLG (Dinamarca, suministros), Agravis (Alemania, suministros), Kerry Group (Irlanda, lácteos).

COOPERATIVAS VACAS LECHERAS (ALTA \% Cuota Mercado y BAJO \% Tasa Crecimiento): Friesland Campina (Holanda, lácteos), Danish Crown (Dinamarca, cárnicos), Vion Food (Holanda, cárnicos), InVivo (Francia, suministros).

COOPERATIVAS INTERROGANTES (BAJA \% Cuota Mercado y ALTO \% Tasa Crecimiento): Tereos (Francia, cultivos y azúcar), Terrena (Francia, multiproducto), Sodiaal (Francia, lácteos), VIVESCIA (Francia, suministros y cereales), Agrial (Francia, multiproducto), Glanbia4 (Irlanda, lácteos), Agrana (Austria, azúcar, almidón y fruta), RWZ Rhein Main, Köln (Alemania, suministros agrarios).

COOPERATIVAS PERROS (BAJA \% Cuota Mercado y BAJO \% Tasa Crecimiento): DMK (Alemania, lácteos), Metsä Goup (Finlandia, forestal), FloraHolland (Holanda, horticultura), Lantmännen (Suecia, multiproducto), Axéréal (Francia, suministros agrarios), Danish Agro (Dinamarca, suministros agrarios), Agricola tre valli (Italia, cárnicos), RWA (Austria, suministros agrarios).

\section{b) Resultados de la Matriz BCG, año 2013:}

COOPERATIVAS ESTRELLAS (ALTA \% Cuota Mercado y ALTO \% Tasa Crecimiento): Bay Wa (Alemania, suministros), Friesland Campina (Holanda, lácteos), Arla Foods (Dinamarca, lácteos), DLG (Dinamarca, suministros), Danish Crown (Dinamarca, cárnicos).

COOPERATIVAS VACAS LECHERAS (ALTA \% Cuota Mercado y BAJO \% Tasa Crecimiento): Agravis (Alemania, suministros), Vion Food (Holanda, cárnicos), InVivo (Francia, suministros), Kerry Group (Irlanda, lácteos).

COOPERATIVAS INTERROGANTES (BAJA \% Cuota Mercado y ALTO \% Tasa Crecimiento): DMK (Alemania, lácteos), Agrial (Francia, multiproducto), Axéréal (Francia, suministros agrarios), Danish Agro (Dinamarca, suministros agrarios), RWZ Rhein Main, Köln (Alemania, suministros agrarios).

COOPERATIVAS PERROS (BAJA \% Cuota Mercado y BAJO \% Tasa Crecimiento): Metsä Goup (Finlandia, forestal), Tereos (Francia, cultivos y azúcar), Terrena (Francia, multiproducto), Sodiaal (Francia, lácteos), FloraHolland (Holanda, horticultura), VIVESCIA (Francia, suministros y cereales), Lantmännen 
(Suecia, multiproducto), Glanbia4 (Irlanda, lácteos), Agricola tre valli (Italia, cárnicos), Agrana (Austria, azúcar, almidón y fruta), RWA (Austria, suministros agrarios).

Un primer resultado, a destacar, es que la mayoría de las cooperativas TOP25 analizadas corresponden a los Modelos Nórdico y Centroeuropeo siguiendo el criterio de Baamonde (2009), como era de esperar, de acuerdo con su dimensión y volumen de negocio mayor. Solamente aparece una cooperativa italiana (las cooperativas de Italia aparecen globalmente como Modelo Mediterráneo), que se posiciona como Perro (Baja Cuota Relativa de mercado y Bajo Crecimiento empresarial) en los dos años analizados. Un segundo resultado importante es el crecimiento global del grupo TOP25 que se observa en la figura 3: incremento de valor del $8,09 \%$ en 2012 y del 9,57\% en 2013 .

Figura. 3. Evolución de la Cifra de Ventas de las TOP25 Cooperativas Agroalimentarias de la UE-28, años 2011-2013

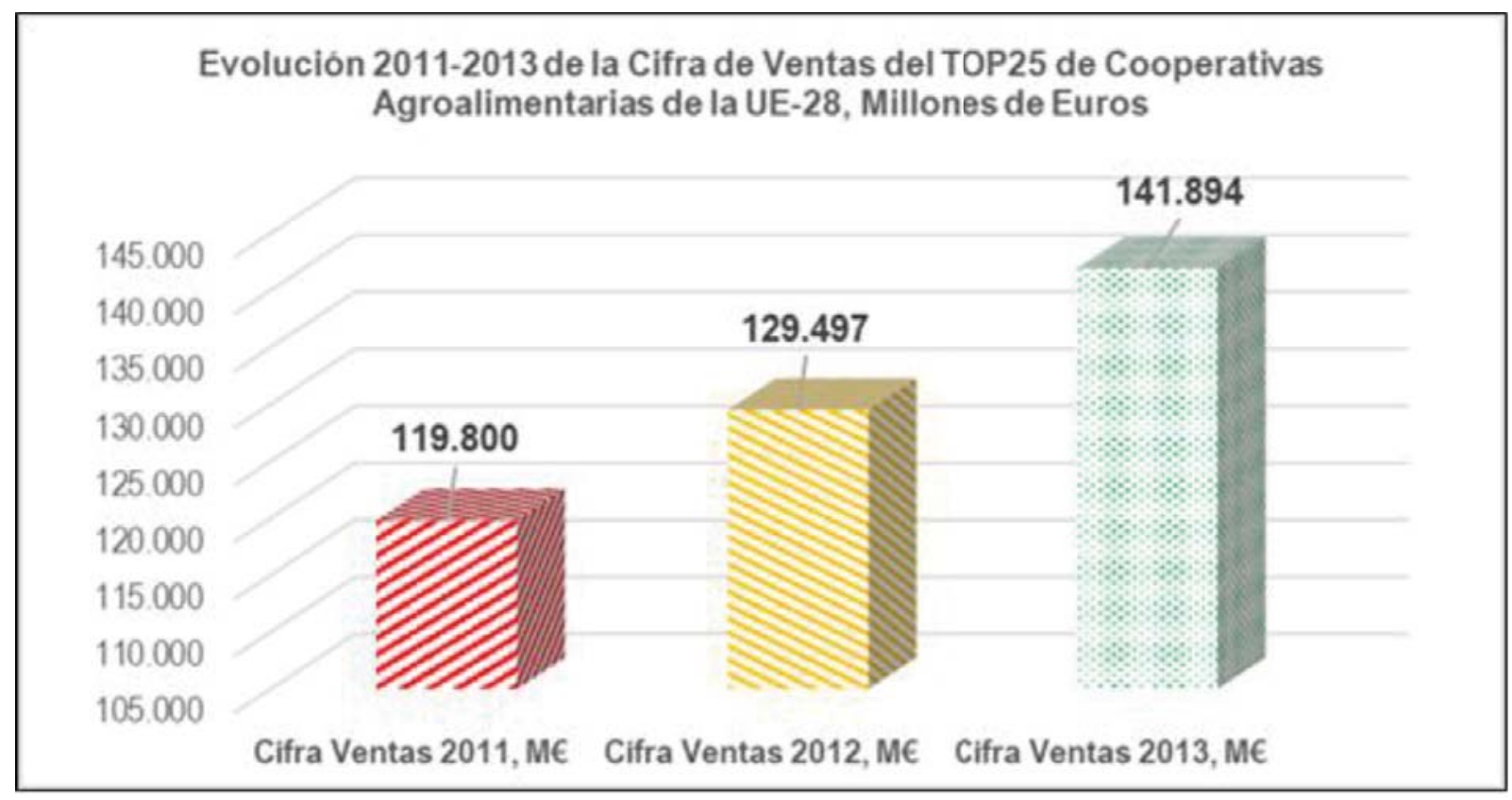

Fuente: Elaboración propia, a partir de datos de COGECA (2015)

En 2012, aparecen como cooperativas mejor posicionadas, es decir como Estrellas (Alta Cuota Relativa de mercado y Alto Crecimiento empresarial) Bay Wa, Arla Foods, DLG, Agravis y Kerry Group. Como Vacas Lecheras (Alta Cuota Relativa de mercado y Bajo Crecimiento empresarial) están posicionadas Friesland Campina, Danish Crown, Vion Food e InVivo. En relación a las cooperativas Interrogantes (Baja Cuota Relativa de mercado y Alto Crecimiento empresarial) aparecen un grupo mayoritario de cinco cooperativas francesas que son Tereos, Terrena, Sodiaal, VIVESCIA y Agrial, a las que se añaden la irlandesa Glanbia4, la austriaca Agrana, y la alemana RWZ Rhein Main, Köln. Respecto las cooperativas Perros (Baja Cuota Relativa de mercado y Bajo Crecimiento empresarial), están en este grupo en 2012 DMK, Metsä Goup, FloraHolland, Lantmännen, Axéréal, Danish Agro, Agricola tre valli y RWA. 
En 2013, las cooperativas Bay Wa, Arla Foods y DLG consolidan su buena posición de Estrellas, y se añaden Friesland Campina y Danish Crown (que en 2012 estaban clasificadas como Vacas Lecheras debido al bajo crecimiento desde 2011 a 2012), migrando a Vacas Lecheras las cooperativas Agravis y Kerry Group. Dentro de las Vacas Lecheras, aparte de las dos anteriores, se consolidan dentro del grupo la holandesa Vion Food y la francesa InVivo. Dentro de las cooperativas Interrogantes solo permanecen dos de las que aparecen en 2012: la francesa Agrial y la alemana RWZ Rhein Main, Köln; las demás han migrado: la alemana DMK, la francesa Axéréal y la danesa Danish Agro las tres desde Perros, mejorando así al menos su crecimiento empresarial desde 2012 a 2013. Como cooperativas Perro, con baja cuota relativa y bajo crecimiento empresarial, permanecen la finlandesa Metsä Goup, la hortícola holandesa FloraHolland, la sueca multiproducto Lantmännen, la cárnica italiana Agricola tre valli y la austriaca de suministros agrarios RWA; en cambio han migrado desde la posición de Interrogantes a Perros (posicionándose con un \% bajo de tasa de crecimiento) las cooperativas francesas Tereos, Terrena, Sodiaal y VIVESCIA, la irlandesa Glanbia4 y la austriaca Agrana.

\section{Análisis BCG del grupo TOP10 de las cooperativas agroalimentarias de España, años 2015-16}

En este apartado, se presenta el análisis BCG del grupo TOP10 global (incluye sociedades cooperativas de $1^{\circ}$ y de $2^{\circ}$ grado) con mayor cifra de ventas en el año 2016. En la tabla 4 se muestran los datos sobre este conjunto de sociedades cooperativas.

Los citados datos de las Cooperativas Agroalimentarias españolas TOP10 del periodo 2015-2016 obtenidos de Cooperativas Agro-alimentarias $(2017,2018)$ han sido procesados con hoja de cálculo Excel, obteniendo los resultados que se exponen en la tabla 4 y la figura 4.

El tratamiento o proceso de cálculo ha consistido, como en el caso del punto 4 anterior, en la determinación de las dos variables \% Cuota Relativa de Mercado y $\%$ de Cuota o Tasa de Crecimiento en el Mercado, para cada una de las sociedades cooperativas españolas TOP10 analizadas. En la penúltima columna "Clase de Empresa 2016" aparecen los resultados de clasificación y posicionamiento en Estrellas, Interrogantes, Vacas Lecheras y Perros, para el año 2016, que genera el gráfico Excel de la figura 4, correspondiente. 
Tabla. 4. TOP 10 de las Cooperativas Agroalimentarias españolas. Datos y resultados para el análisis BCG 2016

\begin{tabular}{|c|c|c|c|c|c|c|c|c|c|c|c|}
\hline $\mathbf{N}^{0}$ & Cooperativa & CC.AA. & Grado & $\begin{array}{l}\mathrm{N}^{0} \\
\text { Socios }\end{array}$ & Empleados & $\begin{array}{l}\text { Cifra } \\
\text { de } \\
\text { Ventas } \\
2015 \\
\text { (M/€) }\end{array}$ & $\begin{array}{l}\text { Cifra } \\
\text { de } \\
\text { Ventas } \\
2016 \\
\text { (M/€) }\end{array}$ & $\begin{array}{l}\% \text { Cuota } \\
\text { Relativa } \\
\text { TOP10 } \\
2016\end{array}$ & $\begin{array}{l}\% \text { Cuota } \\
\text { Crecimiento } \\
2015 \text { a } 2016\end{array}$ & $\begin{array}{l}\text { Clase de } \\
\text { Empresa } \\
\text { BCG } \\
2016\end{array}$ & $\begin{array}{l}\text { Actividades } \\
\text { cooperativizadas }\end{array}$ \\
\hline 1 & DCOOP & ANDALUCÍA & 2 & 208 & 520 & 937 & 1.163 & $20,22 \%$ & $24,12 \%$ & ES & $\begin{array}{l}\text { Aceite de oliva, Vino, } \\
\text { Aceituna de mesa, } \\
\text { Suministros, Servicios } \\
\text { y Exportación. }\end{array}$ \\
\hline 2 & COREN & GALICIA & 2 & 10 & 3.240 & 950 & 1.000 & $17,39 \%$ & $5,26 \%$ & VL & $\begin{array}{l}\text { Piensos, Avícola, } \\
\text { Vacuno de carne, } \\
\text { Porcino, Quesos, } \\
\text { Leche, Servicios y } \\
\text { Exportación. }\end{array}$ \\
\hline 3 & GRUPO AN & NAVARRA & 2 & 159 & 1.260 & 716 & 767 & $13,34 \%$ & $7,12 \%$ & ES & $\begin{array}{l}\text { Cul. herbáceos, } \\
\text { Avicola, Hortalizas, } \\
\text { Carburantes, Inputs, } \\
\text { Frutas, Porcino, } \\
\text { Piensos, Exportación, } \\
\text { Prod. ecológicos, Sec. } \\
\text { de crédito, Tiendas y } \\
\text { Servicios. }\end{array}$ \\
\hline 4 & $\begin{array}{l}\text { SAT } \\
\text { CENTRAL } \\
\text { LECHERA } \\
\text { ASTURIANA }\end{array}$ & $\begin{array}{l}\text { PRINCIPADO } \\
\text { DE } \\
\text { ASTURIAS }\end{array}$ & 1 & 7.470 & 17 & 708 & 673 & $11,70 \%$ & $-4,94 \%$ & VL & $\begin{array}{l}\text { Leche de vaca y } \\
\text { Servicios. }\end{array}$ \\
\hline 5 & ANECOOP & $\begin{array}{l}\text { COMUNIDAD } \\
\text { VALENCIANA }\end{array}$ & 2 & 68 & 209 & 614 & 663 & $11,53 \%$ & $7,98 \%$ & ES & $\begin{array}{lr}\text { Cítricos, } & \text { Frutas, } \\
\text { Hortalizas, } & \text { Vino, } \\
\text { Exportación } & \text { y } \\
\text { Productos ecológicos. }\end{array}$ \\
\hline 6 & COVAP & ANDALUCÍA & 1 & 7.301 & 690 & 415 & 405 & $7,04 \%$ & $-2,41 \%$ & PE & $\begin{array}{l}\text { Piensos, Vacuno de } \\
\text { carne, Ovino de carne, } \\
\text { Porcino, Leche, } \\
\text { Suministros, Servicios, } \\
\text { Sección crédito y } \\
\text { Exportación. }\end{array}$ \\
\hline 7 & ACOR & $\begin{array}{l}\text { CASTILLA y } \\
\text { LEÓN }\end{array}$ & 1 & 4.470 & 541 & 312 & 323 & $5,62 \%$ & $3,53 \%$ & PE & $\begin{array}{lr}\text { Azúcar, } & \text { Cultivos } \\
\text { herbáceos } & \text { y } \\
\text { Suministros. } & \end{array}$ \\
\hline 8 & COBADU & $\begin{array}{l}\text { CASTILLA y } \\
\text { LEÓN }\end{array}$ & 1 & 9.753 & 171 & 275 & 272 & $4,73 \%$ & $-1,09 \%$ & PE & $\begin{array}{l}\text { Vacuno de carne, } \\
\text { Ovino de leche, } \\
\text { Porcino, Leche, } \\
\text { Quesos, Suministros, } \\
\text { Servicios y Tiendas. }\end{array}$ \\
\hline
\end{tabular}




\begin{tabular}{|l|l|l|l|l|l|l|l|l|l|l|l|}
9 & AGROPAL & $\begin{array}{l}\text { CASTILLA } \\
\text { LEÓN }\end{array}$ & 1 & 7.294 & 392 & 253 & 260 & $4,52 \%$ & $2,77 \%$ & PE & $\begin{array}{l}\text { Cereales, Forrajes, } \\
\text { Piensos, Suministros, } \\
\text { Leche, } \begin{array}{l}\text { Quesos, } \\
\text { Vacuno, etc. }\end{array}\end{array}$ \\
\hline 10 & JAENCOOP & ANDALUCÍA & 2 & 11 & 17 & 177 & 225 & $3,91 \%$ & $27,12 \%$ & $? ?$ & Aceite de oliva. \\
\hline
\end{tabular}

Fuente: Cooperativas Agro-alimentarias $(2017,2018)$ y elaboración propia (ES=Estrella; ??=Interrogante; VL=Vaca Lechera; $\mathrm{PE}=$ Perro). En la columna " $\mathrm{N}^{\circ}$ Socios" aparece el número de socios "personas físicas" en las cooperativas de primer grado y el número de socios "personas jurídicas" en las de segundo grado

Figura. 4. Matriz BGC de las TOP10 Cooperativas Agroalimentarias españolas, año $2016^{5}$

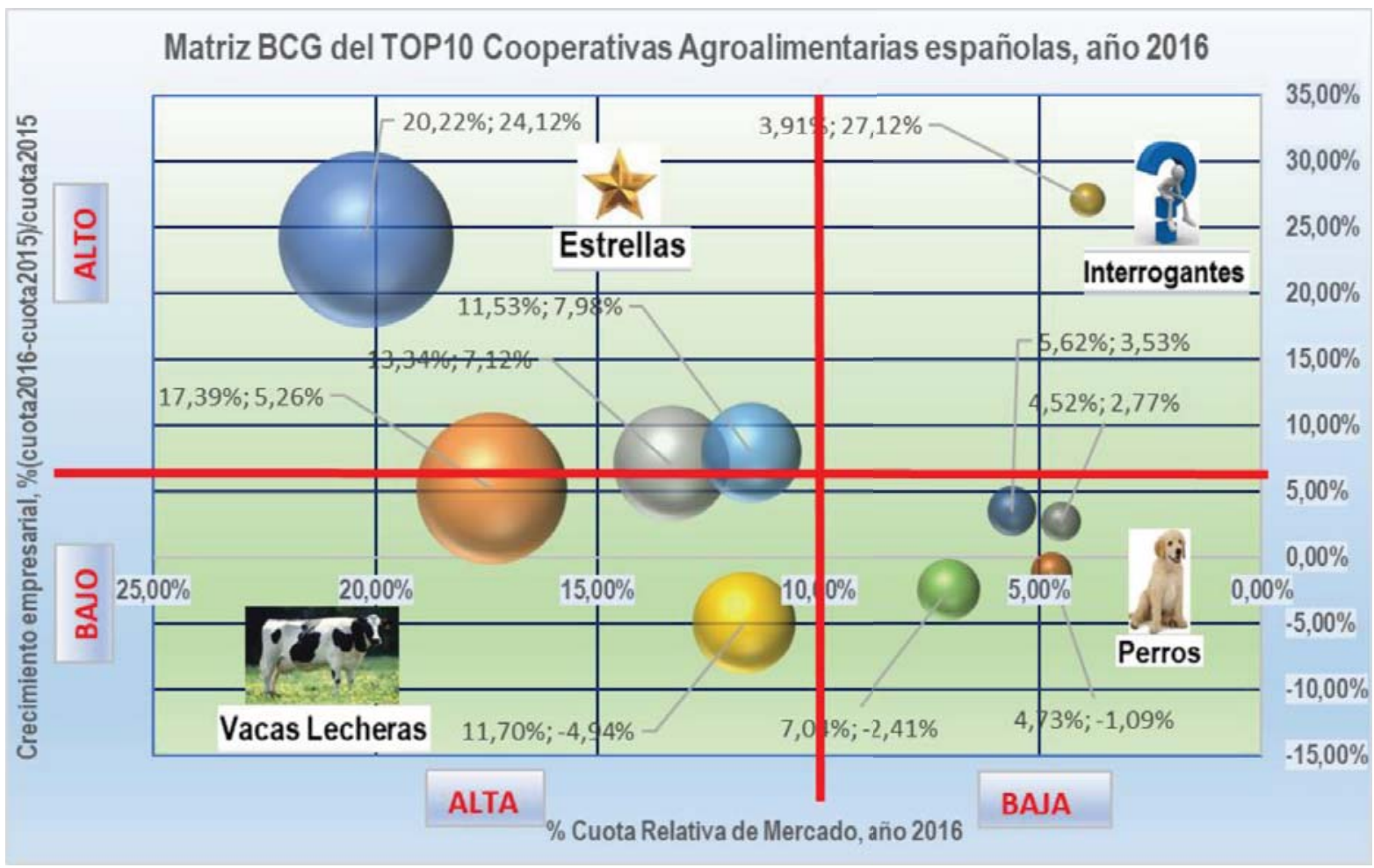

Fuente: Elaboración propia a partir de datos de Cooperativas Agro-alimentarias, 2017, 2018

Antes de realizar el Análisis BCG, será interesante perpetrar la comparación de este grupo TOP10 de cooperativas agroalimentarias españolas con las del TOP25 europeas analizadas anteriormente. En primer lugar, se puede señalar como ya se ha citado antes, que la mayoría de las cooperativas TOP25 europeas pertenecen a los Modelos Nórdico y Centroeuropeo según criterio de Baamonde (2009), con

5 Nota 2: En el gráfico se muestran para cada una de las TOP10 cooperativas agroalimentarias españolas los valores de las variables \% Cuota Relativa Mercado y \% Cuota de Crecimiento (crecimiento empresarial), señalando el centro de cada burbuja cooperativa, que es proporcional a dicha cuota relativa de mercado. Con estos datos se identifica la Cooperativa de que se trata por referencia a los datos de la Tabla 4. 
arreglo a su dimensión y volumen de negocio mayor. Simplemente aparece una cooperativa italiana que, en general, la mayor parte de cooperativas de Italia pertenecen al Modelo Mediterráneo. Sin embargo, el grupo del TOP10 de cooperativas españolas, de acuerdo con los mismos indicadores, claramente forman parte del colectivo del Modelo Mediterráneo, según criterio de Baamonde (2009).

En segundo lugar, y relacionado con lo anotado en el párrafo anterior, se remarca que así como la cifra de ventas del TOP25 de cooperativas europeas se mueve entre los 4 y 5 dígitos enteros de Millones de Euros (desde 2.657 Millones de Euros de RWA de Austria a los 15.957 Millones de Euros de Bay Wa de Alemania), en cambio en el TOP10 de cooperativas españolas varía entre los 3 y los 4 dígitos enteros de Millones de Euros (desde los 225 Millones de Euros del grupo cooperativo JAENCOOP de Andalucía a los 1.163 Millones de Euros de DCOOP también de Andalucía).

A partir de los resultados obtenidos aplicando el modelo de Matriz BCG se pueden efectuar los siguientes análisis, discusiones y diagnósticos:

\subsection{Resultados de la Matriz BCG del TOP10 Cooperativas Agroalimentarias españolas, año 2016}

COOPERATIVAS ESTRELLAS (ALTA \% Cuota Mercado y ALTO \% Tasa Crecimiento): Aparecen como Estrellas tres cooperativas de segundo grado que han destacado en los últimos años o incluso décadas por su estímulo, motivación y convencimiento de crecimiento, integración, reorganización empresarial e innovación: se trata de DCOOP en Andalucía, del Grupo AN en Navarra y ANECOOP en la Comunidad Valenciana. Este posicionamiento de liderazgo en el mix de Cuota de Mercado y Crecimiento empresarial premia dicha buena actitud, estrategia y acción de mejora a lo largo de los últimos tiempos de las citadas cooperativas españolas.

COOPERATIVAS VACAS LECHERAS (ALTA \% Cuota Mercado y BAJO \% Tasa Crecimiento): En este análisis aparecen dos Vacas Lecheras: COREN en Galicia, cooperativa de segundo grado, y la SAT Central Lechera Asturiana en el Principado de Asturias. Se remarca que en el caso de COREN, existe un \% de Cuota de Crecimiento empresarial muy cercano a la media, por lo tanto, dicha cooperativa está en la frontera entre Vaca Lechera y Estrella, que se debe tener en cuenta atendiendo al itinerario de estrategia integradora y buena organización empresarial que sigue la citada empresa. En efecto, el Grupo COREN que engloba 10 cooperativas de primer grado y unos 3.200 ganaderos, se ha preocupado en sus 55 años de existencia (el 55 aniversario se cumplió el 10 de septiembre de 2018) en programar una salida a los mercados internacionales aparte del doméstico, con la necesaria expansión productiva. En cuanto a la SAT Central Lechera Asturiana, aunque en el año 2015 la Comisión Nacional de los Mercados y la Competencia (CNMC) le imputó con una multa de 698.477 Euros por conductas anticompetitivas, y diferentes empresas lácteas fueron sancionadas en este mismo expediente por tener un plan acordado sobre el reparto del aprovisionamiento de leche cruda desde el año 2000 hasta 2013, en los últimos años, pese a las dinámicas difíciles del sector productivo lácteo, respecto a costes y precios, dicha empresa ha 
sabido componer una buena estrategia de marketing (ha sido galardonada con un Premio Nacional de Marketing) y genera suficiente valor añadido para un conjunto de más de 3.300 ganaderos con más de 100.000 vacas lecheras. En el cómputo de las campañas de los años 2015 a 2016 disminuyó su facturación pasando de 708 a 673 Millones de Euros, de ahí su posicionamiento como Vaca Lechera.

COOPERATIVAS INTERROGANTES (BAJA \% Cuota Mercado y ALTO \% Tasa Crecimiento): En este análisis BCG aparece solamente aparece como Perro una única cooperativa de segundo grado que es el Grupo JAENCOOP que engloba a 16 cooperativas de primer grado situadas en el centro y sureste de la Provincia de Jaén en Andalucía. Una de ellas, la Cooperativa de Nuestra Señora del Pilar en Villacarrillo (Jaén) tiene la capacidad molturadora de olivas más grande del mundo con $92.000 \mathrm{~kg} /$ hora con sus instalaciones de elaboración de aceite de oliva. Dicho grupo JAENCOOP, aunque computa la menor Cuota Relativa dentro del TOP10 de cooperativas españolas del 3,91\%, en cambio presenta el mayor \% de Cuota de Crecimiento de 2015 a 2016 con un 27,12\%, lo que demuestra su posicionamiento actual hacia la integración y el crecimiento de cuota de mercado.

COOPERATIVAS PERROS (BAJA \% Cuota Mercado y BAJO \% Tasa Crecimiento): Dentro de este posicionamiento se encuentran cuatro cooperativas de primer grado: COVAP en Andalucía y las otras tres ACOR, COBADU y AGROPAL en la Comunidad de Castilla y León. Las cuatro tienen en común su baja Cuota Relativa de mercado dentro del TOP10 global de cooperativas españolas. También, su baja Cuota de Crecimiento empresarial, que en caso de COVAP y COBADU es negativa $(-2,41 \%$ y $-1,09 \%$, respectivamente) en referencia a las campañas de los años 2015 y 2016. Aunque la Cooperativa Ganadera del Valle de los Pedroches (COVAP) aparezca en este posicionamiento en las campañas analizadas, hay que avanzar que los resultados de ejercicios posteriores prometen mejorar el crecimiento empresarial, gracias a las inversiones por valor de 8,2 Millones de Euros en 2017 y 2018, y la previsión de un 9\% de crecimiento de las ventas. La organización y participación en distintos eventos como Jornadas Técnicas, Encuentros empresariales, Ferias Agro-ganaderas y su especial inquietud y dinamismo innovador, prevén un buen futuro para dicha entidad, que en febrero de 2018 ha obtenido el IV Premio de Innovación Agroalimentaria otorgado por el Grupo Joly y Banco de Santander. Por otro lado, COBADU una cooperativa castellana y leonesa que extiende sus sedes a las provincias de Zamora, Salamanca, Valladolid, Ávila y Cáceres (esta última de Extremadura), tiene como principal eje de actividad, la fabricación de piensos compuestos para las explotaciones ganaderas de los cerca de 10.000 socios, que ha seguido desde su creación en el año 1982. No obstante, la propia dinámica agroganadera ha forzado la aparición de otros servicios agrarios. Como en el caso de la anterior, sus expectativas de crecimiento son reales de cara al futuro. Respecto ACOR, Sociedad Cooperativa General Agropecuaria con sede en Valladolid, que tiene el azúcar (tanto de remolacha como de caña) como producto estrella propio, aparte de los servicios agronómicos y de soporte a cultivos, tienen también visos de futuro crecimiento. Finalmente, AGROPAL o Agropecuaria Palentina SCL, se 
preocupa por mejorar sus servicios agrarios y crecer en la venta de alimentos producidos.

\section{Estudio de la gobernanza cooperativa en Europa y España}

Tanto desde la óptica capitalista como de la economía social, se debe aceptar la gran evolución acaecida en el mundo cooperativo europeo desde los puntos de vista de las tendencias a la integración y concentración vertical y horizontal, de la concentración de poder, de la innovación y cambio tecnológico, de la dimensión y estructuración financiera, de los modelos de crecimiento interno y externo, pero sobre todo de la estructura societaria y organizacional, comprendiendo la diversificación y profesionalización del gobierno interno, y la formación de grupos mixtos mercantiles y de economía social. Otra cuestión importante es como la Unión Europea apoya al asociacionismo agrario a través de la PAC y las Organizaciones de Productores (de las cuales una mayoría son cooperativas).

Tal como expresa Carnicer (2017) en su Tesis Doctoral: los sistemas de gobierno interno suponen un pilar fundamental en cualquier organización y más aún si cabe en las cooperativas. No en vano, diversos estudios a nivel europeo han corroborado que la incorporación de innovaciones en las estructuras de gobierno interno de las cooperativas agroalimentarias, comportan una mejora en su rendimiento.

A nivel europeo y en España, en las dos últimas décadas se han ido realizando bastantes estudios e investigaciones sobre el marco estructural y organizativo de las sociedades cooperativas como empresas que son, profundizando cada vez más en los aspectos del gobierno interno, la relación de la estructura organizativa y operativa con sociedades mercantiles, la profesionalización de la administración y el control, y otros aspectos como los inherentes al género y la incidencia y respeto medioambientales. En relación con ello, podemos referenciar los trabajos de Arcas (2001), Arcas y Hernández (2003), Arcas et al. (2000, 2011 a 2011b), Baamonde (2009), Bijman et al. (2013, 2014a, 2014b), Borguen (2004), Borjabad (2001), Carnicer (2017), Chaddad e Iliopoulos (2013), Colom y Mallada (2010), Colom (2015), Hansen et al. (2002), Juárez et al. (2012), Juliá et al. (2004, 2011, 2013), Meliá et al. (2016), Mínguez et al. (2010), Puentes et al. (2009), Salazar y Galve (2009), Valentinov (2004), Volkers y Lees (1996).

Una gran mayoría de autores afirma que el gobierno interno de una sociedad cooperativa es un pilar fundamental y da lugar en grado superlativo a sus éxitos y buenos resultados. En atención al gobierno interno de las sociedades cooperativas, Bijman et al. (2013) han estudiado, analizado y planteado las innovaciones como inductoras de los distintos modelos de gobierno interno. Al final se puede producir la conocida ruptura entre propiedad y control o administración y son muchas las posibles razones que inducen a ello.

Es probable que, en el caso de las grandes sociedades cooperativas o también en las medianas o incluso en algunas pequeñas, se produzcan operatorias estructurales que animen su crecimiento externo (fusiones, absorciones, participaciones en capital social, captación de inversores externos, creación de subsidiarias, configuraciones mixtas o híbridas sociedad cooperativa-sociedad mercantil, etc.). 
Todo ello repercute en lo positivo a la profesionalización y eficiencia del control administrativo de las sociedades cooperativas, aunque puedan existir cuotas de descooperativización (o desmutualización), que pueden amortiguarse mientras se sigan cumpliendo los principios y valores cooperativos, y que por lo tanto se dé más importancia a las personas y la sociedad, que al capital. Bijman et al. (2014a, $2014 b$ ) aducen una serie de innovaciones ${ }^{6}$ que van apareciendo en las sociedades cooperativas en su tránsito de crecimiento y profesionalización, y que son comentadas por Carnicer (2017).

Chaddad e Iliopoulos (2013), proponen distintos modelos de gobierno interno en las cooperativas agroalimentarias del norte y del sur de Europa. En el sur de Europa prevalece el modelo tradicional o el tradicional extendido (este último en sociedades cooperativas de mayor dimensión), mientras que en el norte de Europa se observan transiciones hacia los modelos tradicional extendido, management y corporación. A continuación, se resumen cada uno de estos modelos.

a. El modelo tradicional de gobierno interno es el adoptado por buena parte de las sociedades cooperativas de menor tamaño de los países de la zona mediterránea (sur de la Unión Europea) y está integrado normalmente por la Asamblea General y el Consejo Rector, siendo en algunos países forzoso la existencia de un Consejo Supervisor que es responsable de supervisar y controlar las actividades y decisiones del Consejo Rector, siendo los estatutos de cada sociedad cooperativa los que deben determinar su obligatoriedad, sus funciones y responsabilidades. La elección del Consejo Supervisor la hace la Asamblea General y está compuesto usualmente por miembros de la sociedad cooperativa, aunque pueden existir miembros externos. Ver figura 5, a).

b. El modelo tradicional extendido contiene los órganos de gobierno usuales (la Asamblea General, el Consejo Rector y el Consejo Supervisor, si así se ha concretado), a los que se incorpora una Dirección Profesional que lleva a cabo la gestión o administración de la sociedad cooperativa. Ver figura 5, b).

6 Bijman et al. (2014), señalan siete innovaciones, como son: el nombramiento de gerentes profesionales que lleven a cabo las tareas ejecutivas del Consejo Rector, la introducción del voto proporcional, la presencia de expertos externos en el Consejo Rector y/o en el Consejo Supervisor, la separación legal entre cooperativa asociación y cooperativa empresa, la inclusión de Consejos de Socios (asimilables en el ordenamiento español a las Asambleas Generales de Delegados), y la creación de estructuras de propiedad hibrida, con diferentes tipos de socios (miembros capitalistas e inversores externos). 
Figura. 5. Modelos de gobierno interno cooperativo del Norte y del Sur de Europa

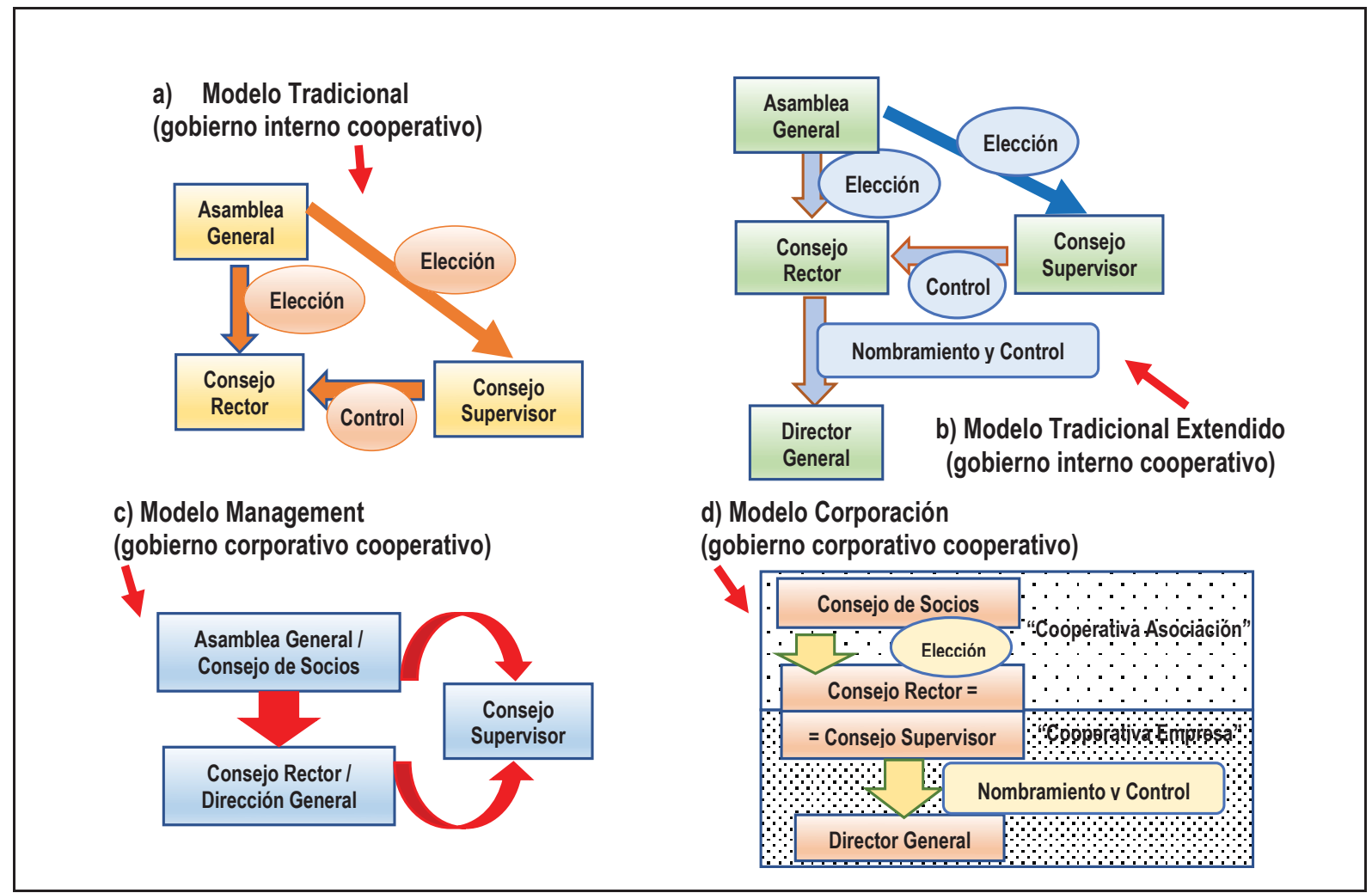

Fuente: Elaboración propia a partir de Carnicer (2017), y de Bijman et al. (2013, 2014a)

c. En el modelo management la caracterización esencial es que el Consejo Rector se ha profesionalizado y ya no está compuesto por miembros de la sociedad cooperativa, sino por gerentes profesionales. Con ello ya no hay una distinción entre decisión y ejecución. Dicho modelo se considera excepcional existiendo en países como Estonia y Países Bajos. Ver figura 5, c).

d. El modelo corporación se formaliza en sociedades cooperativas con separación legal entre la cooperativa asociación y la cooperativa empresa (ver figura 5, d)). Son sociedades cooperativas que han fundado una sociedad de capital (la cooperativa empresa), a la que se le han transferido los activos y la mayoría de las actividades de la cooperativa, transmutando la cooperativa asociación en una sociedad holding que posee el $100 \%$ de las acciones de la sociedad de capital (subsidiaria), aunque este \% puede ser menor. Con este modelo gran parte de las cooperativas de mayor tamaño de los Países Bajos, han trasferido sus actividades comerciales a sociedades de capital, pero han retenido la propiedad cooperativa. Se remarca que la separación legal entre la sociedad de capital y la cooperativa asociación tiene la ventaja de reducir el pasivo, dadas las posibilidades y mayores facilidades de acceso al capital que se consigue, y existe más margen de libertad para los gerentes profesionales de la empresa. 
Tras el resumen de los anteriores modelos, que se pueden encontrar en mayor detalle y extensión en Carnicer (2017), diremos que en el caso de España, según Julià et al. (2011): las cooperativas agroalimentarias de nuestro país se caracterizan por ser muchas y de reducido tamaño y con elevado arraigo local. El modelo español de gobierno interno cooperativo es básicamente el Tradicional (Carnicer, 2017), en el que normalmente existen los tres órganos corporativos: Asamblea General, Consejo Rector e Intervención, en que este último se asimila a un comité de auditoría de las sociedades de capital. No obstante, intermediando modelos de crecimiento interno y externo, concentración e integración vertical y horizontal e internacionalización, se van desarrollando en España modelos de corte híbrido u otros, con tendencia a emular los modelos europeos (sobre todo en la profesionalización ejecutiva y de control).

Tal como se expresa en Juárez y Colom (2018), se puede poner el ejemplo de la empresa INDULLEIDA S.A. una sociedad mercantil promovida y creada por sociedades cooperativas y SAT para transformar frutas y hortalizas y que cuenta con una participación accionarial del $82 \%$ de sociedades cooperativas y SAT $(61 \%$ y $21 \%$ respectivamente), y el $18 \%$ restante de sociedades mercantiles y empresas individuales. En este caso, en el Consejo Delegado de la entidad existe una gran mayoría cooperativa que rige, plantea y practica los principios y valores cooperativos. En la figura 6 se resume la estructura y participación accionarial de la citada empresa.

Figura. 6. Tipología de accionariado y modelo participativo en la empresa INDULLEIDA S.A.

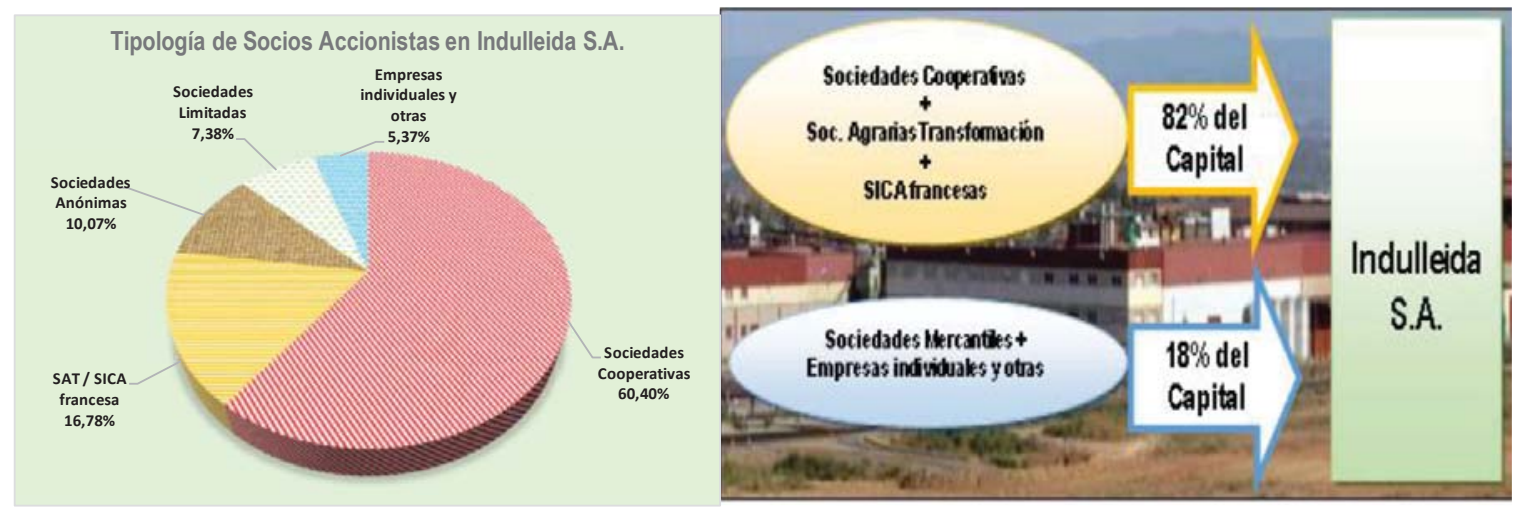

Fuente: Elaboración propia a partir de Juárez F., Colom A. (2018)

Otro ejemplo de modelo híbrido en nuestro país, referenciado en Colom y Juárez (2018), se puede observar en el caso de la Corporación Alimentaria de Guissona S.A. (CAGSA, o bonÀrea en su nombre símbolo-marca actual) que en un principio era la Cooperativa Agropecuaria de Guissona, constituida en 1959, a la que se agregó la Caja Rural de Guissona, cooperativa de crédito instituida en 1963. Al final del anterior milenio, en concreto en diciembre de 1999, se constituyó la CAGSA siguiendo un modelo de crecimiento de diversificación en conglomerado, mediando concentración e integración vertical y horizontal, y sobre todo gran innovación tecnológica y organizativa. 
Hoy día, la Cooperativa Agropecuaria de Guissona sigue funcionando y está integrada en el Grupo empresarial bonÀrea AGRUPA, cuya figura jurídica es la Corporación Alimentaria de Guissona S.A. (CAGSA). La estructuración del accionariado de dicho grupo empresarial se muestra en la figura 7 , y se explica como sigue:

- $\quad$ La Cooperativa Agropecuaria de Guissona posee un $16 \%$ de las acciones de CAGSA;

- La Cooperativa de Crédito Caixa de Guissona posee el 2\% de las acciones de CAGSA;

- $\quad$ Existe una autocartera por valor del 4\% de las acciones; y

- $\quad$ El 78\% restante de las acciones están en propiedad de socios ganaderos y empleados, donde se debe destacar que un $70 \%$ de dicho paquete accionarial es propiedad de socios que lo son también de la Cooperativa Agropecuaria.

Por supuesto, se siguen globalmente los principios y valores cooperativos en la marcha y gestión del grupo empresarial CAGSA.

Figura. 7. Estructura participativa accionarial del grupo empresarial Corporación Alimentaria de Guissona S.A.

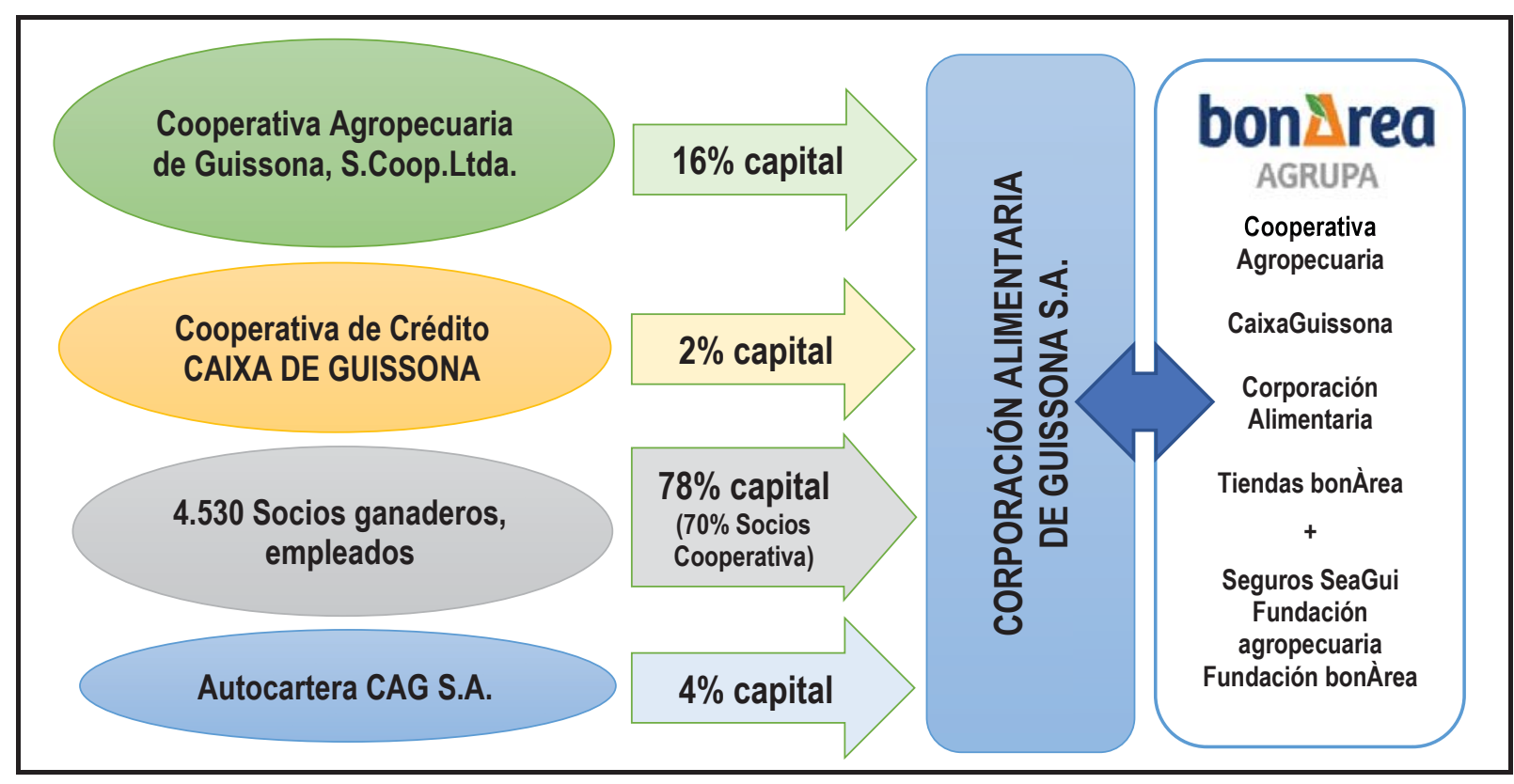

Fuente: Elaboración propia a partir de Colom A., Juárez F. (2018)

\section{Nota final para unas conclusiones}

La Economía Social y el Mundo Cooperativo han ido evolucionando al son de la innovación en la dinámica organizacional y de la gobernanza empresarial, tecnológica, de los modelos de crecimiento interno y externo y de la globalización competitiva y del comercio. No obstante, se han sabido mantener los principios y valores cooperativos, y la política fundamental que induce a que las personas sean más importantes que el capital, a la par que se va tomando más en conciencia el 
papel de los entes empresariales para conservar el medio ambiente y proteger la biodiversidad.

Se ha observado, se ha investigado y analizado en los últimos tiempos, los necesarios cambios y modelos de nuevo diseño para el logro de una resiliencia (resistencia adaptativa) de cara a las nuevas necesidades e impactos del actual entorno globalizador, las nuevas tecnologías TIC, de transporte, de marketing y comercio, y de financiación de las empresas. Para ello, efectivamente algo ha tenido que cambiar en los modelos de gobernanza, para poder adaptar el modus operandi cooperativo a los citados retos consolidados en este nuevo milenio.

Por un lado, la Unión Europea, el papel de soporte y de lobby a la vez de COPA-COGECA, y las Federaciones Nacionales Cooperativas (Cooperativas Agro-alimentarias en el caso español), están actuando como elemento protector y de incentivo para promover y consolidar las transferencias tecnológicas necesarias, disponer de condiciones favorables y tratar de superar los problemas que van surgiendo en el sector, para encaminar bien su solución y el buen futuro (good will). Por otro lado, las Universidades y los Centros de Investigación relacionados con las mismas y que se dedican al mundo cooperativo, están haciendo su papel, tratando de alargar el conocimiento y la tecnología, y por supuesto buscando siempre la explicación y las consecuencias o proyecciones estratégicas hacia un futuro, que se espera mejor. En una tercera vertiente, el Sector Cooperativo, está mutando al modo de adaptarse a las nuevas situaciones cambiantes del macroentorno y la globalización, y para ello debe, por un lado seguir la pauta de la integración y concentración vertical y horizontal, realizar operatorias estructurales (fusiones, absorciones, alianzas estratégicas, etc.) para ganar poder de negociación y dimensión, y establecer una dinámica de elección del mejor sistema o modelo de gobernanza interna y relacional en el clúster correspondiente para poder organizarse, efectuar un mejor control y generar mejores resultados. Por otra parte, se debe abordar y adoptar los cambios tecnológicos y de conocimiento que claramente vayan a solucionar deficiencias, errores o a solucionar problemas sectoriales, y para ello está el polinomio tecnológico $\mathrm{I}+\mathrm{D}+\mathrm{T}+\mathrm{i}$ (el primer binomio de generación de conocimiento y nuevas tecnologías, y el segundo de transferencia e innovación para las empresas). Todo esto es la asignatura pendiente, que van asumiendo y aprobando las sociedades cooperativas españolas.

Se destaca como conclusión, la dinámica adaptativa de los modelos de gobernanza interna citados en este trabajo. Hay que reconocer la propuesta de modelos diferentes de gobierno interno en las cooperativas agroalimentarias del norte y del sur de Europa. En el sur de Europa y por lo tanto en España, prevalece el modelo tradicional o el tradicional extendido (este último en sociedades cooperativas de mayor dimensión), mientras que en el norte de Europa se observan transiciones hacia los modelos tradicional extendido, management y corporación, echando más mano en los modelos híbridos, aduciendo una mayor flexibilidad en las operaciones de gestión empresarial, en la financiación, en la producción y el comercio.

Sería una buena referencia y punto de mira futuro en el caso de nuestro país, tratar de adaptar modelos de crecimiento y gobernabilidad interna cooperativa del norte de Europa, como ya se está haciendo. En el final del punto 6 sobre gobernanza cooperativa en España y Europa anterior, se han explicado dos casos 
españoles: INDULLEIDA S.A. y la Corporación Alimentaria de Guissona S.A., que siguen patrones de modelos mixtos sociedad cooperativa-sociedad mercantil, encontrando soluciones de agilidad, flexibilidad y buena gestión socioeconómica global (productiva y comercial), a la vez que medioambiental, y simultáneamente se asume tajantemente al completo, los principios y valores cooperativos. Son muchos los autores que plantean como favorables estos modelos de corte híbrido, con tendencia a emular los modelos del norte de Europa, sobre todo en la profesionalización ejecutiva y de control, buscando mayor grado de dimensión, poder de negociación, internacionalización y eficiencia empresarial, sin dejar de practicar los citados principios y valores cooperativos, y la asunción del respeto y conservación medioambiental y protección de la biodiversidad.

\section{Referencias bibliográficas}

Arcas, N.; Hernández, M. y Munuera, A. (2000) La relación entre las cooperativas agrarias de primer y segundo grado desde la perspectiva del marketing de relaciones y redes: propuesta de un modelo para su estudio; CIRIEC-España. Revista de Economía Pública, Social y Cooperativa, № 36, pp. 179-202.

Arcas, N. (2001) Proceso de formación y desarrollo de la relación entre las cooperativas agrarias de primer y segundo grado; REVESCO. Revista de Estudios Cooperativos, Primer Cuatrimestre, $\mathrm{N}^{\mathrm{o}} 73$, pp. 7-23.

Arcas, N. y Hernández, M. (2003) Co-ordination and performance of Spanish second-level agricultural co-operatives: The impact of relationship characteristics, en European Review of Agricultural Economics, $\mathrm{N}^{\circ} 30$, pp. 487-507.

Arcas, N. y Hernández, M. (2010) Caso Alimer: Ganar competitividad a través de la fusión; en Munuera Alemán, J. L., coord.: Casos de éxito de las empresas murcianas. Madrid, ESIC; pp. 273-286.

Arcas, N., Alcón, F., Cegarra J.G., Hernández M., López E.I., Marcos G., Martín J.F., Mínguez A. y Tantius P. (2011a) El gobierno de las cooperativas agroalimentarias. Factores de éxito. Fundación Cajamar, Colección Economía (15), 114 pp., Almería, 2011

Arcas, N., Alcón, F., Marcos, G. y López, E. I. (2011b) Las cooperativas agrarias en la Unión Europea y España: evolución de su tamaño y poder de negociación; Tierras de Castilla y León: Agricultura, № 176, pp. 70-79.

Baamonde, E. (2009) El cooperativismo agroalimentario; en Lamo de Espinosa, J., coord.: Mediterráneo Económico (15): El nuevo sistema agroalimentario en una crisis global. Fundación Cajamar, Almería 2009; pp. 229-246.

Bijman, J., Hendrikse, G. y Van Oijen, A. (2013) Accommodating Two Worlds in One Organisation: Changing Board Models in Agricultural Cooperatives. Managerial and Decision Economics, № 34, pp. 204-217.

Bijman, J., Hanisch, M. y Van Der Sangen, G. (2014a) Shifting control? The changes of internal governance in agricultural cooperatives in the EU. Annals of Public and Cooperative Economics, № 85, Vol. 4, pp. 641-661.

Bijman, J., Pyykkönen P. y Ollila P. (2014b) Transnationalization of Agricultural Cooperatives in Europe, The Dovenschmidt Quarterly, No 4, pp. 168-178.

Borgen, S. O. (2004) Rethinking incentive problems in cooperative organizations; en Journal of Socio-Economics, № 33, pp. 383-393. 
Borjabad Gonzalo, P. (2001) El sistema legislativo español de cooperativas y la Ley 27/1999, Revista de Desarrollo Rural y Cooperativismo Agrario, No 5, pp. 7-30.

Carnicer Andrés, M.P. (2017) Nuevos modelos organizativos de las cooperativas agroalimentarias europeas y españolas: Resiliencia vs Desmutualización. Tesis Doctoral, Universidad Politécnica de Valencia, 215 pág.

Chaddad, F.R. y Iliopoulos, C. (2013) Control rights, governance, and the costs of ownership in agricultural cooperatives. Agribusiness, No 29, Vol. 1, pp. 3-22.

COGECA (2015) Development of Agricultural Cooperatives in the EU, 2014. Confederación General de Cooperativas Agrarias, Bruselas, 2014.

Colom A., Mallada C. (2010) Análisis de la estructura y coordinación relacional entre Stakeholders del Clúster de Frutas dulces en la Región Lleida-Huesca, en España. Revista Internacional Agroalimentaria, $\mathrm{N}^{\mathrm{o}}$ 30, Vol. 16, pp. 95-114, Venezuela. Universidad de los Andes.

Colom Gorgues, A. (2015) Guía básica y ejercicios prácticos para la gestión empresarial. Aplicación multisectorial. Ediciones Universidad de Lleida, Serie Eines, 685 pág.

Colom A., Juárez F. (2018) Grupo Alimentario Guissona: formado por una sociedad cooperativa productora de ganado y una sociedad mercantil que transforma $\mathrm{y}$ comercializa dicha producción; en Meliá, E. (Ed.), El gobierno de las sociedades cooperativas agroalimentarias españolas, Cátedra Cajamar de Economía Social, Universidad Politécnica de Valencia, Valencia, Serie Monografías 33, pp. 171-198.

Cooperativas Agro-alimentarias (2017) Informe 2016. Ed. Cooperativas Agro-alimentarias de España. Madrid 2017.

Cooperativas Agro-alimentarias (2018) Informe 2017. Ed. Cooperativas Agro-alimentarias de España. Madrid 2018.

Hansen, M. H., Morrow, J. L. y Batista, J. C. (2002). The Impact of Trust on Cooperative Membership Retention, Performance, and Satisfaction; en International Food \& Agribusiness Management, (5, 1), pp. 41-59.

Henderson, B. D. (1973) The Experience Curve Reviewed, IV. The Growth Share Matrix of the Product Portfolio. The Boston Consulting Group, Perspectives, № 135, Boston, MA, 1973.

Juárez F., Colom A. y Trilla J.L. (2012) ACTEL: una cooperativa de cooperativas con estrategia de crecimiento y mejora competitiva; en el libro: Tamaño y competitividad. Experiencias de crecimiento en las cooperativas agroalimentarias españolas, Cátedra Cajamar, Universidad de Cartagena, Serie Economía, 20, Cartagena diciembre 2013, pp. 227-248.

Juárez F. y Colom A. (2018) INDULLEIDA: una sociedad mercantil promovida por cooperativas y con mayoría de accionariado cooperativo; en Meliá, E. (Ed.), El gobierno de las sociedades cooperativas agroalimentarias españolas, Cátedra Cajamar de Economía Social, Universidad Politécnica de Valencia, Valencia 2018, Serie Monografias 33, pp. 199-220.

Juliá, J.F., Server, R.J. y Meliá, E. (2004). Los procesos de fusión en cooperativas agrarias. Manual de procedimiento. CCAE, FECOAV y Ediciones Mundi Prensa, pp. 145.

Juliá, J.F., Meliá, E. y García, G. (2011) Strategies developed by leading EU agri-food cooperatives in their growth models, Service Business nº, Vol. 1, pp. 27-46.

Juliá, J.F., Meliá, E. y García, G. (2013) Modelos y casos de éxito del cooperativismo agroalimentario en la Unión Europea. En El papel del cooperativismo agroalimentario en la economía mundial, Mediterráneo Económico, Vol. 24, pp. 139-154.

Meliá, E., Carnicer, M.P. y Juliá, J.F. (2016) El gobierno interno de las mayores cooperativas agroalimentarias españolas y su relación con el rendimiento, REVESCO. 
Revista de Estudios Cooperativos, Segundo Cuatrimestre, No 124, pp. 98-113. DOI: 10.5209/REVE.54924.

Mínguez, A., Martín, J. F. y Arcas, N. (2010) Agency and property rights theories in agricultural cooperatives: Evidence from Spain; en Spanish Journal of Agricultural Research, $(8,4)$, pp. 908-924.

Puentes, R., Velasco, M. y Vilar, J. (2009) El buen gobierno en las sociedades cooperativas; REVESCO. Revista de Estudios Cooperativos, Segundo Cuatrimestre, No 98, pp. 118140.

Salazar, I. y Galve, C. (2009) Ejercicio de las funciones empresariales por parte de los socios en las cooperativas agrarias; REVESCO. Revista de Estudios Cooperativos, Primer Cuatrimestre, $\mathrm{N}^{\mathrm{o}} 101$, pp. 79-106.

Valentinov, V. (2004) Toward a Social Capital Theory of Co-operative Organisation; en Journal of Co-operative Studies, $\mathrm{N}^{\mathrm{o}}$ 37, pp. 5-20.

Volkers, R. y Lees, M. (1996) Corporate governance. and management control systems in European co-operatives. Disponible en http://www.gdrc.org/icm/coop-europe.html (2604-2010). 